\title{
Scaling of Pulsed Nanosecond Capillary Plasmas at Different Specific Energy Deposition
}

\author{
Yifei Zhu ${ }^{1,2}$, Svetlana M. Starikovskaia ${ }^{1}$,Natalia Yu Babaeva ${ }^{3}$ and Mark J. Kushner ${ }^{4}$ \\ 1 Laboratory of Plasma Physics (CNRS, Ecole Polytechnique, Sorbonne Universities, Univ. Paris-Sud), Institut \\ Polytechnique de Paris, F-91128, Palaiseau, France \\ 2 Institute of Aero-engineering, School of Mechanical Engineering, Xi'an Jiaotong University, Xi'an 710049, \\ People's Republic of China \\ 3 Joint Institute for High Temperatures Russian Academy of Sciences, Izhorskaya 13, Moscow 125412, Russia \\ 4 Department of Electrical Engineering and Computer Science, University of Michigan, 1301 Beal Ave., Ann \\ Arbor, Michigan 48109-2122, USA \\ E-mail: yifei.zhu.plasma@gmail.com,svetlana.starikovskaia@lpp.polytechnique.fr, \\ nybabaeva@gmail.com,mjkush@umich.edu
}

\begin{abstract}
Nano-second, capillary discharges (nCDs) are unique plasma sources in their ability to sustain high specific energy deposition $\omega_{\text {dep }}$ approaching $10 \mathrm{eV} /$ molecule in molecular gases. This high energy loading on short timescales produces both high plasma densities and high densities of molecular exited states. These high densities of electrons and excited states interact with each other during the early afterglow through electron collision quenching and associative ionization. In this paper we discuss results from a 2-dimensional computational investigation of a nCD sustained in air at a pressure of 28.5 mbar and with a voltage amplitude $20 \mathrm{kV}$. Discharges were investigated for two circuit configurations - a floating low voltage electrode and with the low voltage electrode connected to ground through a ballast resistor. The first configuration produced a single ionization wave from the high to low voltage electrode. The second produced converging ionization waves beginning at both electrodes. With a decrease of the tube radius, the velocity of the ionization fronts decreased while the shape of the ionization wave changed from the electron density being distributed smoothly in the radial direction, to being hollow shaped where there is a higher electron density near the tube wall. For sufficiently small tubes, the near-wall maxima merge to have the higher density on the axis of the capillary tube. In the early afterglow, the temporal and radial behavior of the $\mathrm{N}_{2}\left(\mathrm{C}^{3} \Pi_{\mathrm{u}}\right)$ density is a sensitive function of $\omega_{\text {dep }}$ due to electron collision quenching. These trends indicate that starting from $\omega_{\text {dep }} \geq 0.3 \mathrm{eV} /$ molecule, it is necessary to take into account interactions of electrons with electronically excited species during the discharge and early afterglow.
\end{abstract}

PACS numbers: 82.33, 52.20, 34.80

Keywords: nanosecond pulsed discharge, capillary discharge, plasma modeling 


\section{Introduction}

Nanosecond pulsed discharges, characterized by high reduced electric fields and strongly nonequilibrium plasmas [1]-[5], are efficient for generating highly reactive species and producing fast gas heating [6], [7]. Production of reactive species is important for surface functionalization and biomedical applications [8]-[15]. Fast gas heating is important to plasma assisted flow control and, together with production of reactive species, for plasma-assisted combustion [16]-[21]. The production of these reactive species is a sensitive function of the manner of energy deposition - specifically the pulse power format and specific energy deposition $\left(\mathrm{J} / \mathrm{cm}^{3}\right)$. Controlling these properties then provides a means to optimize nanosecond pulsed discharges for specific applications.

While nanosecond capillary discharges (nCD) sustained at atmospheric pressure are widely used for biomedical applications, the nCD sustained at moderate pressures [22]-[25] is an attractive tool to investigate fundamental plasma processes as a function of operational parameters, as well as the specific energy deposition. The terms fast ionization wave (FIW) and streamer are usually used when describing propagation plasmas in nCDs. FIW is typically used for moderate pressures (hundreds of mbar) while the term streamers is usually used for atmospheric pressure. Characterization of FIWs includes measurements of the longitudinal electric field by calibrated capacitive probes [23], [26]. These measurements indicate that normalized electric fields, electric field/gas number density, of up to many thousands of Td occur during the few first nanoseconds $[1 \mathrm{Td}$ (Townsend) = $10^{-17} \mathrm{~V} \cdot \mathrm{cm}^{2}$. Although insightful, such measurements typically have some spatial and time averaging, and so are not able to resolve the finer structure of the FIW.

New diagnostics such as E-FISH [27], [28] are approaching the time and spatial scales required to resolve FIW dynamics. However, multi-dimensional numerical simulations currently provide some of the most detailed perspectives of the development of capillary discharges, bolstered by comparison to available experiments. For example, two-dimensional (2D) simulations were performed of a Xe/Ne discharge initiated by a nanosecond high-voltage pulse propagating through a branched channel consisting of two straight inlet and outlet sections separated by a circular loop [29]. Qualitative agreement was obtained with experiments for the predicted dynamics of the FIW splitting, propagation and merging. Streamer propagation in a capillary tube in air at atmospheric pressure was modeled by Jansky et al [30]. The tube radius, relative permittivity and photoionization were found to influence the 2D distribution of plasma parameters. Modeling of a nCD at moderate pressure was performed by Klochko et al [31], where the calculated FIW propagation speed, current rise and $E / N$ rise agreed well with experiments.

When the FIW in a moderate pressure nCD reaches the low voltage electrode, the conductive channel connecting the two electrodes enables a high current density, and as a consequence, high energy deposition. These conditions in turn provide a high density of electrons and electronically excited species. By varying voltage amplitude, tube radius and length, it is possible to adjust the specific energy deposition (SED) in the plasma. Recent measurements by optical emission spectroscopy and two photon absorption laser induced fluorescence (TALIF)of a nCD, with accompanying kinetics modeling [25] [32], have shown that an increase of the SED increases the probability of interaction of excited and/or charged species with each other. That is, the reaction mechanism becomes highly nonlinear with increasing SED and can lead to significantly different outcomes than at low values of SED. The results of 1-dimensional (1D) modeling of a nCD in air by Popov suggested that for SED of $\geq 1 \mathrm{eV} /$ molecule (the following text will use $\mathrm{eV} / \mathrm{mol}$ for simplicity), molecular oxygen is nearly completely dissociated in the early afterglow (1-2 $\mu \mathrm{s})$ [33]. These trends were 
explained by dissociative collisions of $\mathrm{O}_{2}$ with excited nitrogen molecules. Zhu et al. proposed that the optical actinometry method to obtain absolute O-atom densities in the early afterglow of nCD must take into account the change of kinetics at high SED [34].

In this paper, we discuss results from 2D numerical simulations of a nanosecond capillary discharge at moderate pressure. We found that the spatial distribution of the fast ionization wave in nCDs is a sensitive function of tube radius during the propagation breakdown stage. When the FIW produces a conducting channel between the electrodes, the dynamics transition from being centered on the axis of the tube to becoming wall dominated. The discharge is also sensitive to the specific energy deposition during the low-voltage, high current stage following breakdown due to increasing reaction rates between excited species.

Descriptions of the model and operating conditions are given in Section 2. The kinetics scheme and validation are discussed in Section 3. The propagation of the discharge front and the spatial-temporal distribution of species are discussed in Section 4 with the aim of providing insights to the evolution of the plasma at high electric fields over a wide range of SED. Concluding remarks are in Section 5.

\section{Description of the Model and Implementation of Experimental Conditions}

The numerical simulations were performed using nonPDPsim, a hybrid code which uses unstructured meshes. A detailed description of the model can be found in [35]. In this section, we briefly describe the equations solved in model for this investigation.

Continuity equations are solved using a drift-diffusion approximation for the fluxes of charged species, neutral species and electron energy:

$$
\begin{aligned}
& \frac{\partial n_{i}}{\partial t}+\nabla \cdot \boldsymbol{\Gamma}_{\mathbf{i}}=S_{i}, i=1,2, \ldots, N_{t} \\
& \frac{\partial n_{e} \varepsilon}{\partial t}+\nabla \cdot \boldsymbol{\Gamma}_{\boldsymbol{\varepsilon}}=P_{e}-n_{e} \Sigma N_{i} \kappa_{i} \\
& \boldsymbol{\Gamma}_{\mathbf{i}}=-\left(q_{i} /\left|q_{i}\right|\right) \mu_{i} n_{i} \nabla \Phi-D_{i} \nabla n_{i}, i=1,2, \ldots, N_{c} \\
& \boldsymbol{\Gamma}_{\mathbf{i}}=-D_{i} \nabla n_{i}, i=1,2, \ldots, N_{n} \\
& \boldsymbol{\Gamma}_{\boldsymbol{\varepsilon}}=\frac{5}{3} \varepsilon \boldsymbol{\Gamma}_{\mathbf{e}}-\lambda \nabla T_{e}
\end{aligned}
$$

where $\Phi$ is electrical potential, and $n_{i}, \Gamma_{i} D_{i}, \mu_{i}, q_{i}$ and $S_{i}$ are the number density, flux, diffusion coefficient, mobility, charge and source function for species $i$. The source function $S_{i}$ includes gain and loss terms due to gas phase reactions (neutral chemistry, ion-molecule reactions and electron impact processes) and contributions associated with wall chemistry, including electron emission from surfaces. $N_{t}, N_{c}$ and $N_{n}$ are the number of total, charged and neutral species, receptively. $n_{e}$ is the electron number density. $\varepsilon \equiv 3 k_{B} T_{e} / 2, P_{e}, \kappa_{i}, \Gamma_{e}$ and $\lambda$ are the mean electron energy, power absorbed from the electric field by electrons, power lost in collision $i$, electron thermal flux, and ther- 
mal conductivity. The total absorbed power $P_{\varepsilon}$ is computed assuming Joule heating by all charged species:

$$
P_{\varepsilon}=\nabla \phi \cdot \sum_{i=1}^{N_{c}}\left[q_{i}\left(D_{i} \nabla n_{i}+\left(q_{i} /\left|q_{i}\right|\right) \mu_{i} n_{i} \nabla \Phi\right)\right]
$$

Where $q_{i}$ is the charge of species $i$. The thermal conductivity of electrons $\lambda$ is [36]:

$$
\lambda=8 n_{e} T_{e} /\left(\pi m_{e} v_{m}\right)
$$

where $v_{m}$ is the electron momentum transfer collision frequency. The system of equations 1-7 is closed by solving Poisson's equation for the electric potential:

$$
-\nabla(\epsilon \nabla \Phi)=\sum_{i=1}^{N_{c}} q_{i} n_{i}+\rho_{m}
$$

where $\rho_{m}$ is the charge density on surfaces and in materials:

$$
\frac{\partial \rho_{m}}{\partial t}=\sum_{j=1}^{N_{c}} q_{j}\left(-\nabla \cdot \Gamma_{\mathbf{j}}\left(1+\gamma_{j}\right)\right)-\nabla \cdot(\sigma(-\nabla \Phi)) .
$$

$\gamma_{\mathrm{j}}$ is the secondary electron emission coefficient for species $\mathrm{j}$, and $\sigma$ is the conductivity of solid materials.

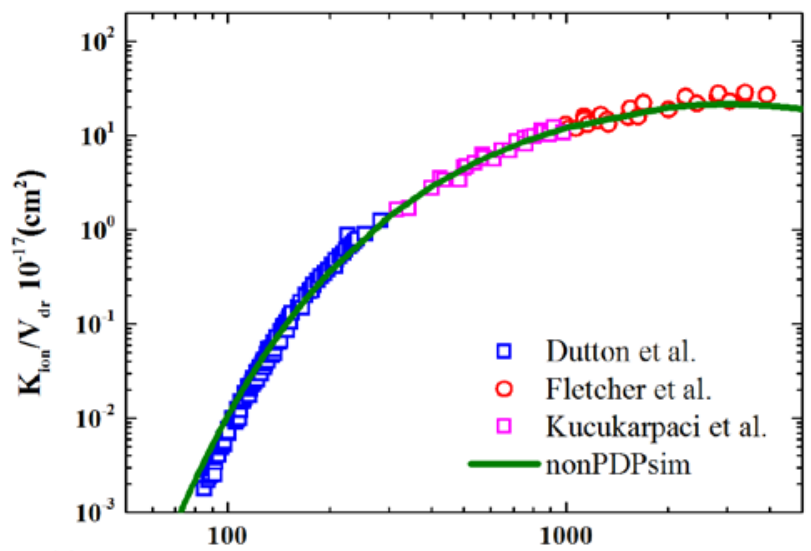

(a)

$\mathbf{E} / \mathbf{N}(\mathbf{T d})$

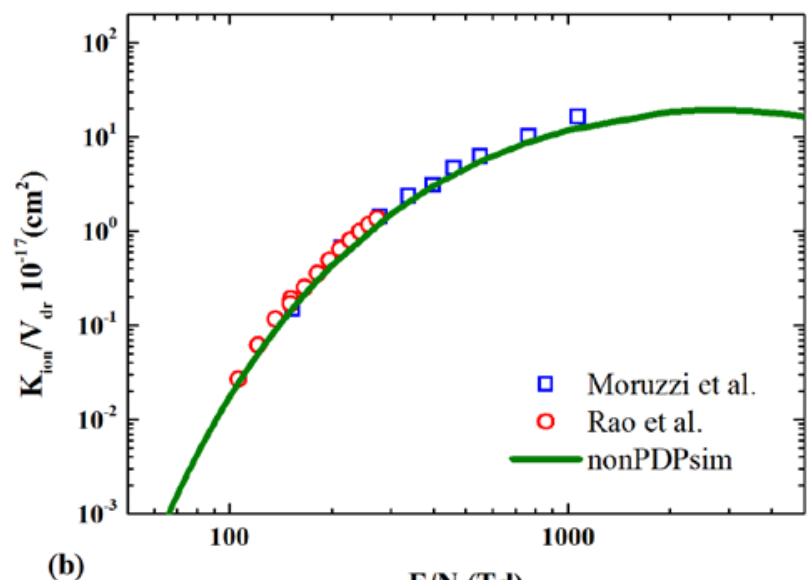

$\mathrm{E} / \mathrm{N}$ (Td)

Figure 1. Validation of computed ionization coefficient of (a) pure $\mathrm{N}_{2}$ and (b) air. Experimental data represented by points are taken (a) from Refs. [37]-[39] and (b) from Refs [40], [41].

The electron transport coefficients and rate coefficients used to solve the equations (1-9) were obtained by solving Boltzmann's equation for the electron energy distribution (EED) using a two-term 
spherical harmonic approximation. Solving Boltzmann's equation over a range of E/N (electric field/gas number density) produced a table of collision frequency and reaction rate coefficients as a function of electron temperature. Given the local electron temperature from the model, rate coefficients were determined by interpolating this table based on the local mean energy approximation. The accuracy of the EED is critical in modeling nCDs. To be consistent with our previous study of nCD [34], the reaction mechanism utilized the following sources:

(i) The electron impact cross sections for $\mathrm{N}_{2}(\mathrm{v}=0-8), \mathrm{N}_{2}\left(\mathrm{~A}^{3} \Sigma_{\mathrm{u}}^{+}\right), \mathrm{N}_{2}\left(\mathrm{~B}^{3} \Pi_{\mathrm{g}}\right), N_{2}\left(\mathrm{~W}^{3} \Delta_{u}\right)$, $\left.\mathrm{N}_{2}\left(\mathrm{~B}^{\prime 3} \Sigma_{\mathrm{u}}^{-}\right), \mathrm{N}_{2}\left(\mathrm{a}^{1} \Pi_{\mathrm{g}}\right), \mathrm{N}_{2}\left(\mathrm{a}^{\prime 1} \Sigma_{\mathrm{u}}^{-}\right), \mathrm{N}_{2}\left(\mathrm{~W}^{1} \Delta_{\mathrm{u}}\right), \mathrm{N}_{2}\left(\mathrm{C}^{3} \Pi_{\mathrm{u}}\right), \mathrm{N}_{2}\left(\mathrm{E}^{3} \Sigma_{\mathrm{g}}^{+}\right), \mathrm{N}_{2}(\operatorname{sum}), \mathrm{O}_{2}(4.5 \mathrm{eV})\right]$ were based on the results of Pitchford and Phelps [42]. Not all these species are considered when solving transport equations and the electron energy equation, but their energy losses are included when solving Boltzmann's equation for the EED.

(ii) Electron impact excitation e $+\mathrm{N}_{2}\left(B^{3} \Pi_{g}\right) \rightarrow e+N_{2}\left(C^{3} \Pi_{u}\right)$ is one of the more important processes in the nCDs discussed in this work. The cross section for this process (and by detailed balance, the reverse reaction) were taken from the work of Bacri and Medani [43].

To confirm that the cross sections used in the simulation reproduce swarm data, the ionization coefficient, $\mathrm{K}_{\text {ion }}$ and drift velocity $\mathrm{V}_{\mathrm{dr}}$ were calculated as a function of $E / N$. The calculated results are favorably compared with experimental data in Figure 1 for nitrogen and air.

Photo-ionization is accounted for using a propagator method described in Ref. [35]. The photo-ionization source for species $\mathrm{m}$ at location $\boldsymbol{r}_{\boldsymbol{i}}$ due to the integral of vacuum ultraviolet (VUV) photon emission from all other locations $\boldsymbol{r}_{\boldsymbol{j}}$ by species $k$ is given by

$$
\begin{gathered}
S_{m}\left(\mathbf{r}_{\mathbf{i}}\right)=\sigma_{k m}^{I} N_{m}\left(\mathbf{r}_{i}\right) A_{k} \int N_{k}\left(\mathbf{r}_{j}^{\prime}\right) G_{k}\left(\mathbf{r}_{j}^{\prime}, \mathbf{r}_{\mathbf{i}}\right) d^{3} \mathbf{r}_{\mathbf{j}}^{\prime} \\
G_{k}\left(\mathbf{r}_{j}^{\prime}, \mathbf{r}_{\mathbf{i}}\right)=\frac{\exp \left(-\int_{\mathbf{r}_{\mathbf{j}}^{\prime}}^{\mathbf{r}_{\mathbf{i}}} \Sigma_{l} \sigma_{l k} N_{l}\left(\mathbf{r}_{n}^{\prime}\right) d \mathbf{r}_{n}^{\prime}\right)}{4 \pi\left|\mathbf{r}_{\mathbf{i}}-\mathbf{r}_{\mathbf{j}}^{\prime}\right|^{2}}
\end{gathered}
$$

where $N_{k}$ is the density of the radiating species having Einstein coefficient $A_{k}, \sigma_{k m}^{I}$ is the photo-ionization cross section for species $m$ by photons emitted by species $k$ and $\sigma_{l k}$ is the total absorption cross section for photon $k$ by species $l . G_{k}\left(\mathbf{r}_{\mathbf{j}}^{\prime}, \mathbf{r}_{\mathbf{i}}\right)$ is Green's function for the survival of the photons emitted at the location $\mathbf{r}_{\mathbf{j}}$ to reach location $\mathbf{r}_{\mathbf{i}}$, and also accounts for view angles and obscuration which may block the radiation. For the $\mathrm{N}_{2} / \mathrm{O}_{2}$ mixture investigated here, resonance radiation from the $\mathrm{N}_{2}$ singlet states is the photo-ionization source in the wavelength range of $98-102.5 \mathrm{~nm}$. The photo-ionization cross section for $\mathrm{O}_{2}$ was parameterized between $\sigma_{k}=0.1-1 \stackrel{\circ}{\mathrm{A}}^{2}$ [44]. By comparing with experimental results (discussed in the following section), the photo-ionization cross section was selected to be $\sigma_{k}=0.6 \stackrel{\circ}{\AA^{2}}$.

In the present work, only transport of charged particles, radicals and photons, and diffusion of neutral species, are addressed. Gas heating and the hydrodynamic motion of the neutral gas resulting from temperature gradients are not taken into account due to the short time scale, the constant tube volume and the fact that few processes of interest at this stage are sensitive to gas temperature.

A description of the experimental setup and the geometry of the capillary discharge are in Refs. [25], [34]. The discharge was initiated in a quartz capillary tube. Two grounded aluminum plates 
enclose the capillary from above and below. Adhesive aluminum sheets form a closed, grounded screen having a rectangular cross section. The discharge tube is terminated by two metal pin-shaped high voltage (HV) and low voltage (LV) electrodes. The HV electrode is connected to a generator that supplies the voltage pulses. The LV electrode is left unterminated and connected to the long cable. A different boundary for the potential of the LV electrode was also considered in the simulations.

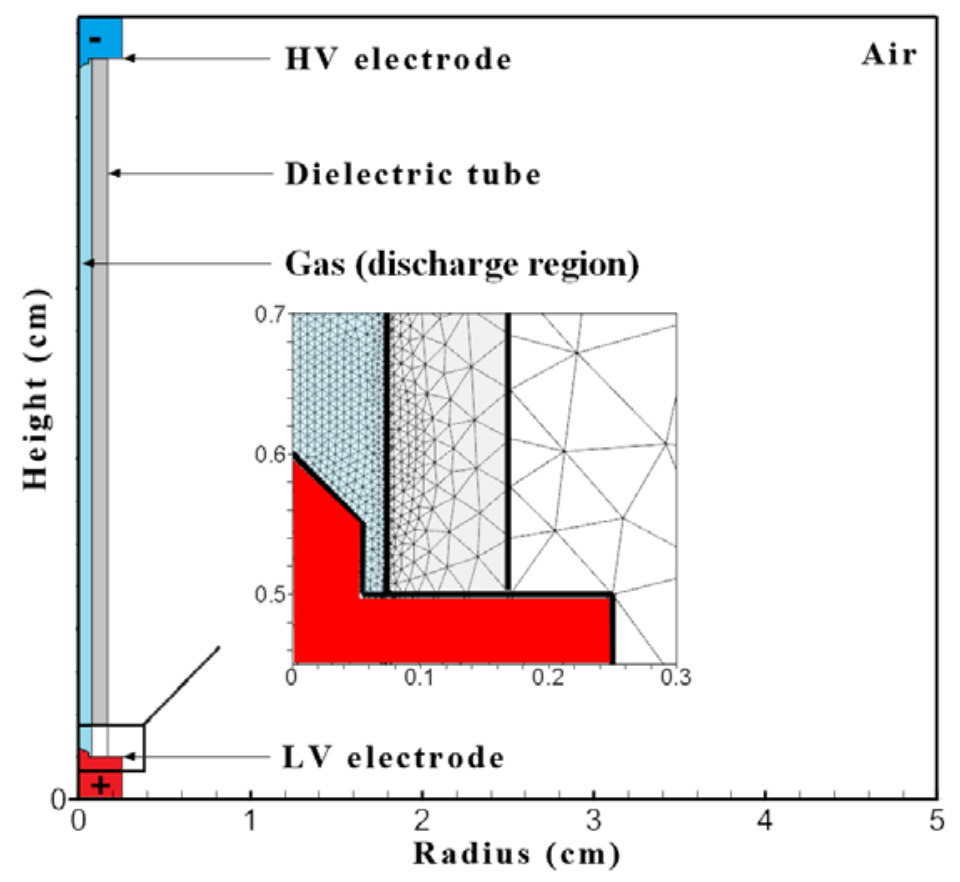

Figure 2. 2D cylindrical geometry for the nCD with an enlargement of the low voltage electrode showing the numerical mesh refinement. The length of tube ranges from $2 \mathrm{~cm}$ to $8 \mathrm{~cm}$.

The simplified 2D cylindrical geometry for used in this work is shown in Figure 2. The geometry consists of a quartz capillary tube with relative permittivity $\varepsilon_{r}=4$. To directly compare to prior experiments [31], the actual discharge tube radius was used in the model. To reduce the computational cost when using a fine numerical mesh and more detailed kinetics, the following changes were implemented:

(i) The length of the capillary tube was reduced from $8 \mathrm{~cm}$ to $L=2 \mathrm{~cm}$. This reduction in length preserves the main features of the discharge while reducing the calculation time for propagation of the discharge. The calculation then focuses more on the behavior of the discharge after closing the discharge gap where the main energy is deposited. The voltage rise time was also reduced to enable a constant value of voltage before the ionization wave closes the gap.

(ii) The radius of the capillary tube for the base case, $\mathrm{R}_{1}=0.75 \mathrm{~mm}$, corresponds to the experiment. To investigate the dependence of discharge phenomena as a function of radius of the discharge tube, calculations were also performed for radii of $\mathrm{R}_{2}=1.5 \mathrm{~mm}$ and $\mathrm{R}_{3}=3.75 \mathrm{~mm}$.

Boundary conditions for the transport equations were assigned according to species and materials. For the transport equations, a flux in/flux out boundary condition was used for all the plas- 
ma-material contacting surfaces. For each species, a "consumption" coefficient $\alpha_{i}$ and "production" coefficient $\beta_{k, i}$ are specified where $\alpha_{i}$ is the probability that species $i$ is consumed by the surface and $\beta_{k, i}$ is the rate of production of species $k$ by species $i$. The returning flux from the surface is then

$$
\Gamma_{r}=\Gamma_{r}^{0}\left(1-\alpha_{r}\right)+\sum_{k} \Gamma_{r}^{0} \beta_{k, r}
$$

where $\Gamma_{r}^{0}$ is the flux into the surface assuming the surface density is zero and the sum is over other species. For ions, $\alpha_{r}$ is 1 and $\beta$ for the corresponding neutral is 1.0. The secondary electron emission coefficient for ions $\beta_{k, r}$ is 0.01 .

For the electron energy equation, an electron temperature of $0.05 \mathrm{eV}$ is assigned to all surfaces in contact with the plasma. This adiabatic boundary condition avoids the possibility of overestimating electron density adjacent to tube wall.

For Poisson's equation, the electric potential boundary conditions on metal surfaces are obtained from a circuit model. Each metal surface is connected to ground through a simple electrical circuit consisting of a resistor and power supply (whose voltage setting would be zero for grounded metals). Currents through each leg of the circuit are obtained by summing charged particle fluxes. Electrically floating metal surfaces are actually represented by dielectrics having sufficiently high conductivity and permittivity that they appear to be equipotential surfaces. Charging of dielectric surfaces is accounted for by computing the surface charge densities from the charged particle fluxes onto the surface as in Equation (9). Photoelectron emission from surfaces was not considered.

The initial electron density was $n_{e 0}=10^{9} \mathrm{~cm}^{-3}$ uniformly distributed in the capillary tube to account for the residual electron density from prior pulses. In addition, a small spot of plasma with a Gaussian spatial distribution was placed at the tip of $\mathrm{HV}$ electrode $(\mathrm{r}=0 \mathrm{~cm}, \mathrm{z}=0.6 \mathrm{~cm}$ ),

$$
\left.n_{e}(r, z)=10^{13} \exp \left(-\left(\frac{r}{0.01 \mathrm{~cm}}\right)^{2}-((z-0.6 \mathrm{~cm}) / 0.01 \mathrm{~cm})^{2}\right)\right) \mathrm{cm}^{-3} \text {. }
$$

The initial ion density is given based on quasi-neutrality. The initial voltage applied to the HV electrode is $3 \mathrm{kV}$ to eliminate the computationally time-consuming voltage rise stage prior to initiation of the discharge.

\section{Kinetics Reduction and Validation}

In our previous work Ref [34], a reduced kinetics scheme of 47 reactions for air with 5.3\% Ar was proposed. The aim was to describe the temporal evolution of the electron density and selected excited species in a nanosecond capillary discharge and to predict the density of oxygen atoms in the ground state. The kinetics scheme was validated with experimental measurements.

Unless otherwise noted, the operating conditions are a pressure of $\mathrm{P}=28.5 \mathrm{mbar}$ (2850 Pascal, 21.4 Torr $)$ and a gas mixture of nitrogen and oxygen $\left(\mathrm{N}_{2} / \mathrm{O}_{2}=80 / 20\right)$. The voltage pulse has a $20 \mathrm{kV}$ amplitude with a rise time of 2 ns, plateau of $6 \mathrm{~ns}$ and decay time of 2 ns. To account for the gas mixture used in this work, this previously derived kinetics scheme in Ref [34] was adjusted.

(i) The original scheme was validated on the basis of the temporal evolution of electron density, and optical emission from $\mathrm{N}_{2}\left(\mathrm{C}^{3} \Pi_{\mathrm{u}}\right), \mathrm{O}\left(3 \mathrm{p}^{3} \mathrm{P}\right)$, and $\operatorname{Ar}\left(2 \mathrm{p}_{1}\right)$. In this work, validation was based on emission from $\mathrm{N}_{2}\left(\mathrm{C}^{3} \Pi_{\mathrm{u}}\right)$.

(ii) A validated kinetics scheme for streamer propagation can be found in Ref [45]. The kinetics takes into account the reactions between charged species and were added to our reduced kinetics scheme in [34].

(iii) Associative ionization reactions are responsible for long-lived plasmas with high specific en- 
ergy deposition. The following reactions were added to the model [33]:

$$
\begin{array}{ll}
\mathrm{N}_{2}\left(\mathrm{~A}^{3} \Sigma_{\mathrm{u}}^{+}\right)+\mathrm{N}_{2}\left(\mathrm{a}^{\prime 1} \Sigma_{\mathrm{u}}^{-}\right) \rightarrow \mathrm{N}_{4}^{+}+\mathrm{e} & \left(\mathrm{k}=3 \times 10^{-12} \mathrm{~cm}^{3} / \mathrm{s}\right) \\
\mathrm{N}_{2}\left(\mathrm{a}^{\prime 1} \Sigma_{\mathrm{u}}^{-}\right)+\mathrm{N}_{2}\left(\mathrm{a}^{\prime 1} \Sigma_{\mathrm{u}}^{-}\right) \rightarrow \mathrm{N}_{4}^{+}+\mathrm{e} & \left(\mathrm{k}=1 \times 10^{-11} \mathrm{~cm}^{3} / \mathrm{s}\right)
\end{array}
$$

where $\mathrm{k}$ is the rate coefficient. Two conditions were used for validation. The first used a short tube $(\mathrm{L}=2 \mathrm{~cm})$ to validate the kinetics scheme by comparing the temporal evolution of $\mathrm{N}_{2}\left(\mathrm{C}^{3} \Pi_{\mathrm{u}}\right)$ with results from a global kinetics model, ZDPlaskin, used in Ref [34] for an actinometry study. The second used a discharge tube commensurate with the experiment $(\mathrm{L}=8 \mathrm{~cm})$ to validate FIW propagation with the results presented in Ref [31].

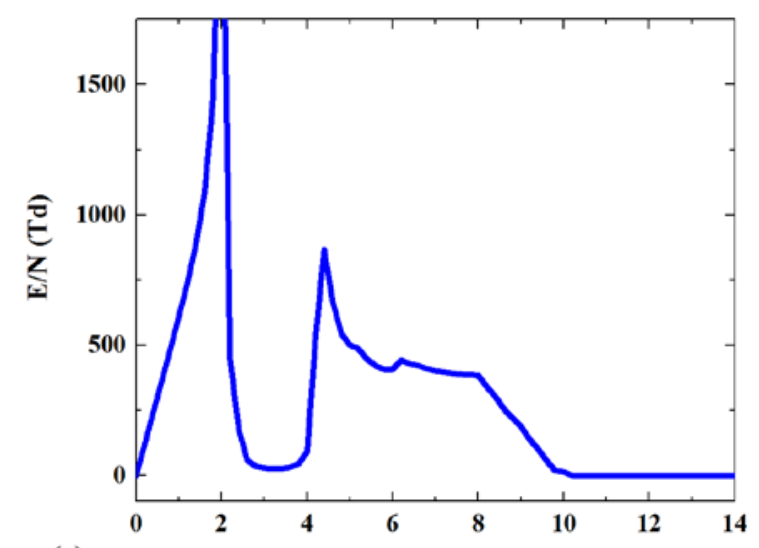

(a)

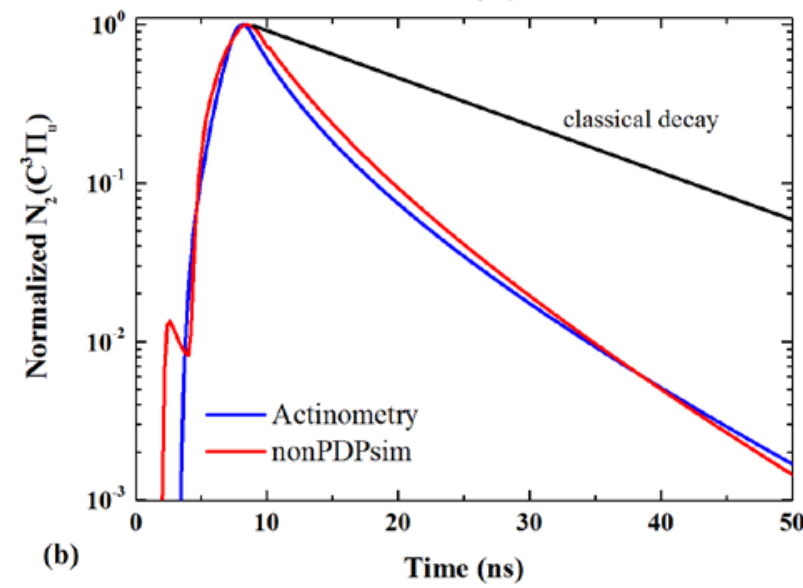

Figure 3. Validation of the reaction mechanism for optical emission from $N_{2}\left(C^{3} \Pi_{u}\right)$. (a) $E / N$ extracted from the $2 \mathrm{D}$ modeling results that were used as input to the global model. (b) Normalized optical emission of $\mathrm{N}_{2}\left(\mathrm{C}^{3} \Pi_{\mathrm{u}}\right)$ predicted by the global model and by nonPDPsim. The classical decay rate is based on only the natural lifetime of $\mathrm{N}_{2}\left(\mathrm{C}^{3} \Pi_{\mathrm{u}}\right)$.

When using the shorter tube, the temporal evolution of $E / N$ and the density of $\mathrm{N}_{2}\left(\mathrm{C}^{3} \Pi_{\mathrm{u}}\right)$ in the middle of the tube were recorded from the 2D simulation, as shown in Figure 3 (a). The $E / N$ profile and the electron density as a function of time from the 2D model were used as input to the global model (without Ar addition) to calculate the density of $\mathrm{N}_{2}\left(\mathrm{C}^{3} \Pi_{\mathrm{u}}\right)$. The time dependence of $\mathrm{N}_{2}\left(\mathrm{C}^{3} \Pi_{\mathrm{u}}\right)$ calculated by nonPDPsim and by the global model, shown in Figure 3(b), are in a good agreement. The classical decay curve represents the decrease in optical emission due only to the natural lifetime of the $\mathrm{N}_{2}\left(\mathrm{C}^{3} \Pi_{\mathrm{u}}\right)$ state, $\sim 42 \mathrm{~ns}$. The optical emission decays with a shorter lifetime, $\sim 10$ ns, due to collisional processes, dominantly with electrons.

The second case, a simulation for the full length of the experiment, provided the axial 
electricpotential as a function of time.These results are compared to experiments [26] in Figure 4 as a function of axial distance starting from the tip of the HV electrode for $t=1-9 \mathrm{~ns}$. In the measurements, the electric potential was measured by moving a capacitive probe along a line parallel to the capillary tube at $r=1.27 \mathrm{~cm}$. The shapes of the potential profiles at the probe location predicted by the model agree well with the scaled experimental profiles. The speed of the ionization wave was determined from the peak electric field deduced from the difference of potential profiles along the axial direction. Both calculations and experiments provide a FIW speed of $\approx 1.4 \mathrm{~mm} \mathrm{~ns}^{-1}$.

\section{Plasma Properties of Capillary Discharges}

The use of FIW in technological devices is motivated by their ability to produce high degrees of ionization, excitation and dissociation. These properties are in large part determined by the spatial distribution of plasma density, which is in turn determined by the spatial distribution of the electric field in the nCD. To investigate these processes, simulations were performed for two types of LV electrode configuration (floating potential and fixed potential) and three radii of the discharge tube.
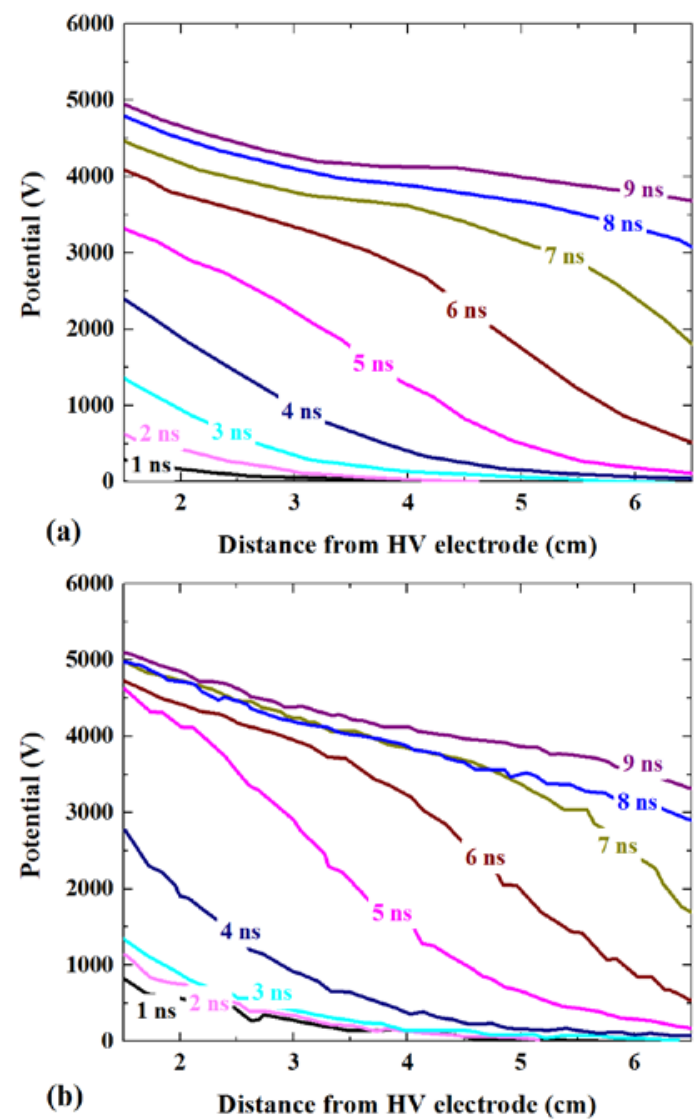

Figure 4. Validation of the axial potential profiles of the FIW during the propagation stage. Results were collected from the position of the tip of a capacitive probe, $r=1.27 \mathrm{~cm}$. (a) Model predictions. (b) Experimental measurements.

\subsubsection{Fast Ionization Wave and Streamer Modes}

In the previous experiments on nCDs [24], [31] which we are simulating, the LV electrode was connected to the central wire of a $100 \mathrm{~m}$ coaxial cable, and the central wire was not physically terminated to ground. In this configuration, the LV electrode is electrically floating. In our simu- 
lations, a "metal" is a material that has a specified electric potential as a boundary condition for solution of Poisson's equation. Dielectrics are materials in which the electric potential is solved for. A floating metal can be approximated by specifying the material to be a dielectric with a high enough conductivity so that there is a negligible electric field inside that material. In the steady state, the potential of the material will be the value that balances the flux of negative and positive charge carriers to its surface - the floating potential. During a transient, the charging time of the material will be inversely proportional to its capacitance.

To contrast these two extremes, two types of LV electrodes were modeled - a "metal like" dielectric that has a high conductivity to simulate a floating metal, and a metal connected to ground through a $100 \Omega$ ballast resistor. The potential on the LV electrode is then the voltage drop across the ballast resistor resulting from current collected by the LV electrode. The radius of the discharge tube is $0.75 \mathrm{~mm}$, the same as in the experiments.

The electron density and $E / N$ in the nCD with an electrically floating LV electrode are shown in Fig. 5. The electron density avalanches to $6 \times 10^{14} \mathrm{~cm}^{-3}$ in the head of the ionization wave during its 2 ns propagation across the tube, with a speed increasing from $0.7 \times 10^{9} \mathrm{~cm} / \mathrm{s}$ to $1.1 \times 10^{9} \mathrm{~cm} / \mathrm{s}$ (or $7 \mathrm{~mm} / \mathrm{ns}$ to $11 \mathrm{~mm} / \mathrm{ns}$ ). The $E / N$ in the head of the ionization wave exceeds $5000 \mathrm{Td}$, producing an ionization rate of $3.6 \times 10^{23} \mathrm{~cm}^{-3} \mathrm{~s}^{-1}$. Due to the LV electrode being electrically floating, a significant voltage drop does not develop between the electrode and the adjacent plasma. As a result, there is little local ionization due to electric field enhancement at the LV electrode to initiate an ionization wave. Ionization is first initiated by electric field enhancement at the HV electrode and then sustained by the space charge producing the large $E / N$ in the head of the ionization wave. This mode of discharge development - a single ionization wave propagating from the $\mathrm{HV}$ to the floating LV electrode - is called the fast ionization wave (FIW) mode.

Once the FIW reaches the LV electrode after $2.4 \mathrm{~ns}$, the tube is filled with a quasi-uniform plasma with an electron density exceeding $10^{15} \mathrm{~cm}^{-3}$ and with a quasi-uniform $E / N$ having a relatively low value (tens of Td). A sheath with a low electron density, $10^{9} \mathrm{~cm}^{-3}$, and high $E / N$ exceeding $12000 \mathrm{Td}$, is formed near the dielectric wall, having a thickness of about $300 \mu \mathrm{m}$. The voltage drop across the sheath is about $2000 \mathrm{~V}$ and the sheath thickness corresponds to about 700 $\lambda_{\mathrm{D}}$ (Debye lengths), as might be expected for floating dielectric in contact with a high plasma density at 28.5 mbar.

When simulating a discharge having a floating LV electrode, a single FIW propagated from the HV electrode to the LV electrode. When simulating a discharge having a LV electrode connected to ground through a ballast resistor, two ionization fronts separately propagated from the HV and LV electrodes towards each other. Although the physics of these ionization waves seems to be the same, we will call the first mode that occurs with a floating LV electrode a "fast ionization wave". The second that occurs with a grounded LV electrode will be called a "streamer" mode in analogy to pin-to-pin atmospheric discharges where two streamers start from the electrodes and merge in the interelectrode space.

The electron density and $E / N$ in the nCD with the LV electrode grounded through a $100 \Omega$ ballast resistor are shown in Fig. 6. In this configuration, ionization waves propagate from both the HV electrode (left side in Fig. 6) and the LV electrode (right side). This is called the "streamer" mode. With the grounded LV electrode, there is a sufficient voltage drop at the tip of the LV electrode, coupled with electric field enhancement, to launch the second ionization wave. The positive streamer propagating left to right from the HV electrode has a lower speed than the FIW 
that occurs with the floating electrode. It takes $1.6 \mathrm{~ns}$ for the positive streamer to propagate $0.5 \mathrm{~cm}$ compared to $1 \mathrm{~ns}$ in the FIW mode. The negative streamer propagating right to left from the LV electrode covers only $0.25 \mathrm{~cm}$ during the same time. However, once formed, the positive streamer accelerates to a higher velocity than the FIW, reaching $1.4 \times 10^{9} \mathrm{~cm} / \mathrm{s}(14 \mathrm{~mm} / \mathrm{ns})$ compared to $1.1 \times 10^{9} \mathrm{~cm} / \mathrm{s}(11 \mathrm{~mm} / \mathrm{ns})$ in the FIW mode.

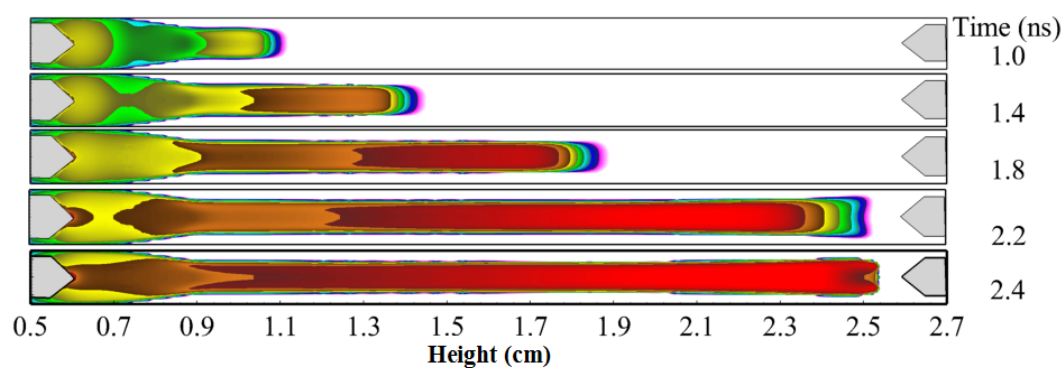

(a) $\mathbf{n}_{\mathbf{e}}$
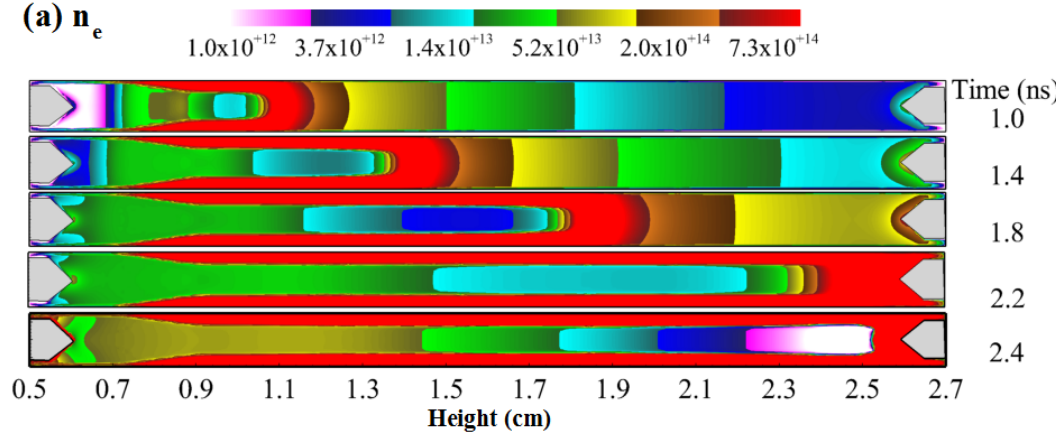

(b) $\mathbf{E} / \mathrm{N}$

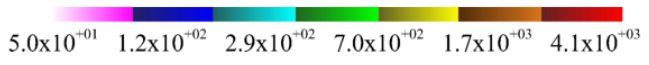

Figure 5. Evolution of an ionization wave in a capillary tube with the LV electrode electrically floating. The HV electrode is at the left and the LV electrode is at the right. This is the FIW mode. (a) electron density in units of $\mathrm{cm}^{-3}$; (b) electric field in units of $\mathrm{Td}\left(10^{-17} \mathrm{~V} \cdot \mathrm{cm}^{2}\right)$. The tube radius is $0.75 \mathrm{~mm}$.

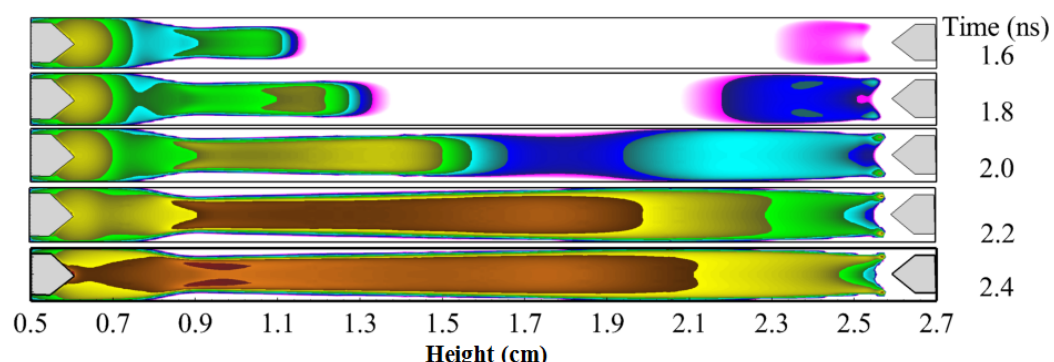

(a) $\mathbf{n}_{\mathrm{e}}$ $\begin{array}{llllll}1.0 \times 10^{+12} & 3.7 \times 10^{+12} & 1.4 \times 10^{+13} & 5.2 \times 10^{+13} & 2.0 \times 10^{+14} & 7.3 \times 10^{+14}\end{array}$

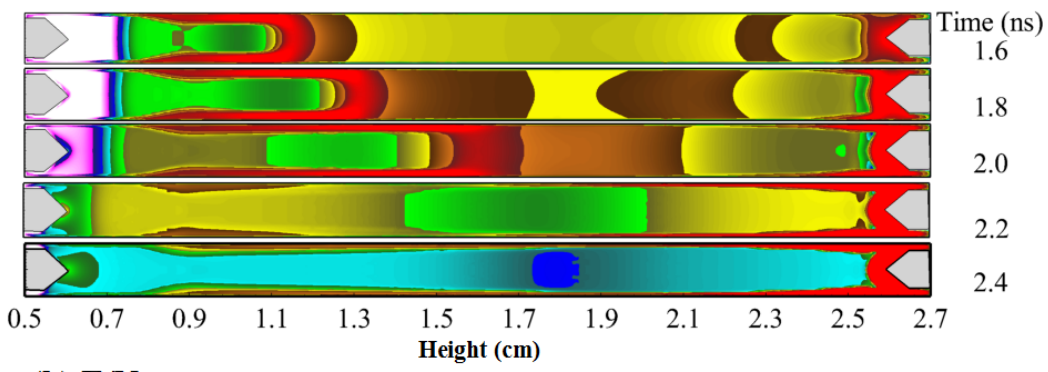

(b) $\mathrm{E} / \mathrm{N}$

$\begin{array}{llllll}1.0 \times 10^{+12} & 3.7 \times 10^{+12} & 1.4 \times 10^{+13} & 5.2 \times 10^{+13} & 2.0 \times 10^{+14} & 7.3 \times 10^{+14}\end{array}$ 
Figure 6. Evolution of ionization waves in a capillary tube with the LV electrode electrically grounded through a ballast resistor. The HV electrode is at the left and the LV electrode is at the right. This is the streamer mode. (a) electron density in units of $\mathrm{cm}^{-3}$. (b) Normalized electric field $(\mathrm{E} / \mathrm{N})$ in units of $\mathrm{Td}\left(10^{-17} \mathrm{~V}-\mathrm{cm}^{2}\right)$. The tube radius is $0.75 \mathrm{~mm}$.

The positive and negative streamers merge at about $2.2 \mathrm{~ns}$, leaving a quasi-uniform plasma between the $\mathrm{HV}$ electrode and the position at which the merger takes place. A lower plasma density extends to the LV electrode. The maximum ionization source in the positive streamer front is $1.5 \times 10^{23} \mathrm{~cm}^{3} \mathrm{~s}^{-1}$ and ionization in the negative streamer is $2.7 \times 10^{22} \mathrm{~cm}^{3} \mathrm{~s}^{-1}$. The thick sheath region near the cathode shields the potential on the LV electrode, leads to a weaker negative ionization wave.

The electron density and $E / N$ on the axis of the FIW and streamer modes are shown in Figure 7. There are several systematic differences between the FIW and streamer modes. (i) The peak electron density and $E / N$ in the FIW mode increase during propagation from the $\mathrm{HV}$ to $\mathrm{LV}$ electrode, reaching $\mathrm{n}_{\mathrm{e}}=10^{15} \mathrm{~cm}^{-3}$ and $E / N=14000 \mathrm{Td}$ at $2.4 \mathrm{~ns}$. This monotonic increase is due to the low $E / N$ (and low voltage drop) behind the streamer in the conductive channel, which then increases voltage ahead of the head of the FIW in the low pre-pulse plasma density. This increased voltage enables a larger $E / N$ in the head of the FIW. In the streamer mode, the increase in the maximum electron density and $E / N$ stops once the two streamers merge, and a conductive path is produced between the electrodes. (ii) The $E / N$ in the head of FIW is 3-4 times higher than that in the head of the streamers. The electron density in the channel of the FIW is also 3-4 times larger than that for the streamers. In spite of these differences, after passage of the ionization waves in both the FIW and streamer modes, the tube is filled with a fairly uniform electron density.

Energy deposition in the nCD takes place dominantly after the propagation of the ionization waves and the tube is filled with plasma. This is true for both the FIW and streamer modes. The $E / N$ at the center of the $\mathrm{nCD}(\mathrm{r}=0 \mathrm{~cm}, \mathrm{z}=1.5 \mathrm{~cm})$ is shown in Fig. 8 for the FIW and streamer modes. In the FIW mode, after a sharp rise and drop in $E / N$ as the ionization front passes ( 2 ns), there is a second peak. This peak results from the voltage drop across the head of the FIW being dissipated by transfer of charge to the electrode, and the voltage being redistributed across the discharge tube. When the ionization wave reaches the LV electrode, the positive charge in the front is neutralized, this leads to the increase of the electric field in the positive channel. Then the electric field drops again after the charge in the channel is also neutralized.

After transit of the ionization front across the tube, the tube is uniformly filled with plasma with a near constant conductivity (after $3 \mathrm{ns).} \mathrm{As} \mathrm{a} \mathrm{result,} \mathrm{the} \mathrm{electric} \mathrm{field} \mathrm{is} \mathrm{largely} \mathrm{uniform} \mathrm{across}$ the tube except for geometrical electric field enhancement at the tips of the electrodes. $E / N$ is then determined by the difference in potentials between the $\mathrm{HV}$ and $\mathrm{LV}$, and their intervening distance. In the streamer mode the $E / N$ in the head of the positive ionization wave head is smaller due to voltage division with the negative ionization wave. However, the end result is the same as for the FIW. Once the tube is filled with uniform plasma, the electric field in the center of the tube is largely determined by the applied voltage and distance between the electrodes (while accounting for electric field enhancement at the electrode tips). The similarities in the uniformity of the plasma and of the electric field after the tube is filled by plasma between the FIW and streamer modes implies that the energy deposition should also be the nearly the same. 

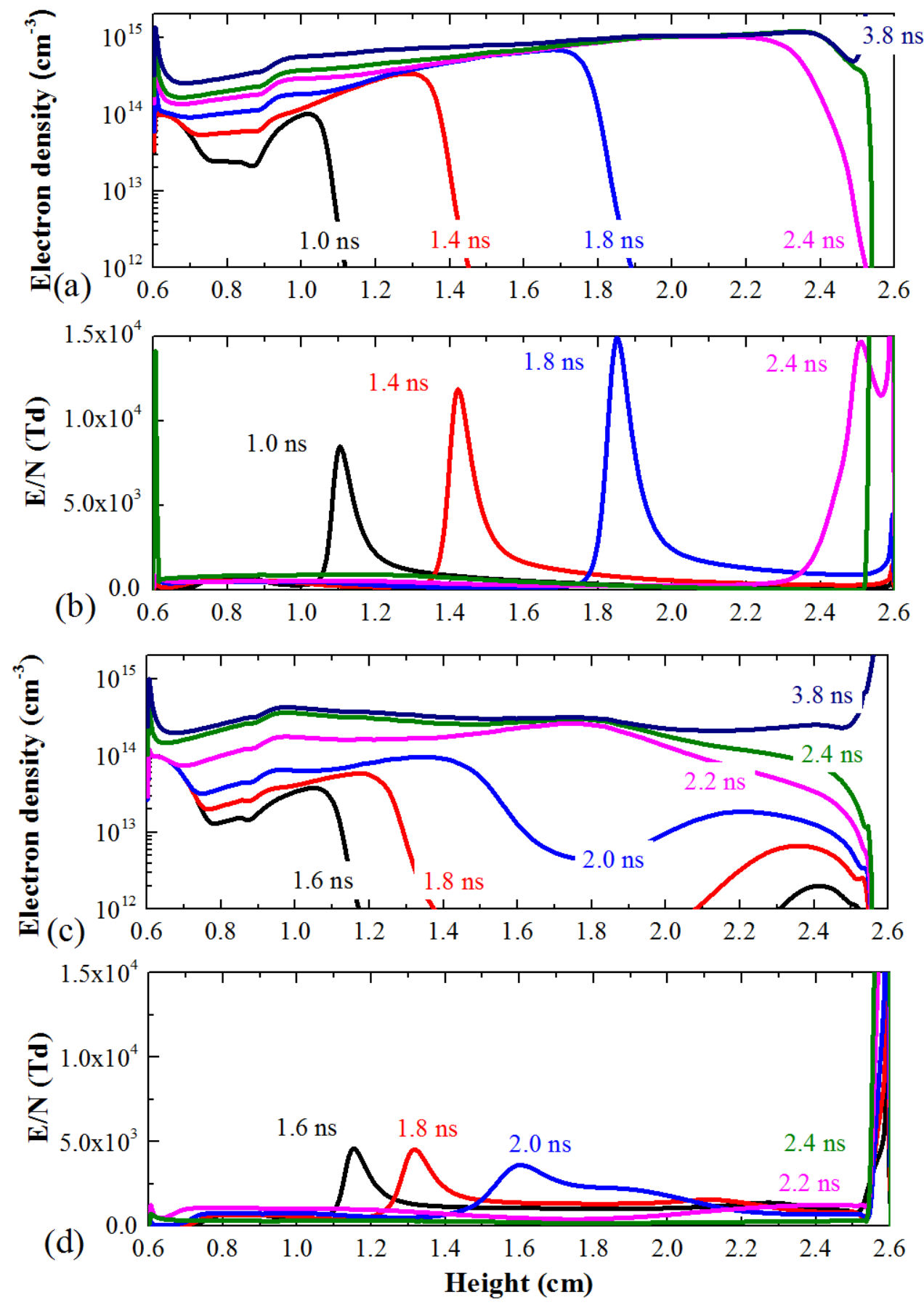

Figure 7. Axial distribution of electron density in (a) the fast ionization wave and (c) the streamer modes; and axial normalized electric field $(\mathrm{E} / \mathrm{N})$ in (b) the fast ionization wave and (d) the streamer modes. The radius of the tube is $0.75 \mathrm{~mm}$. 


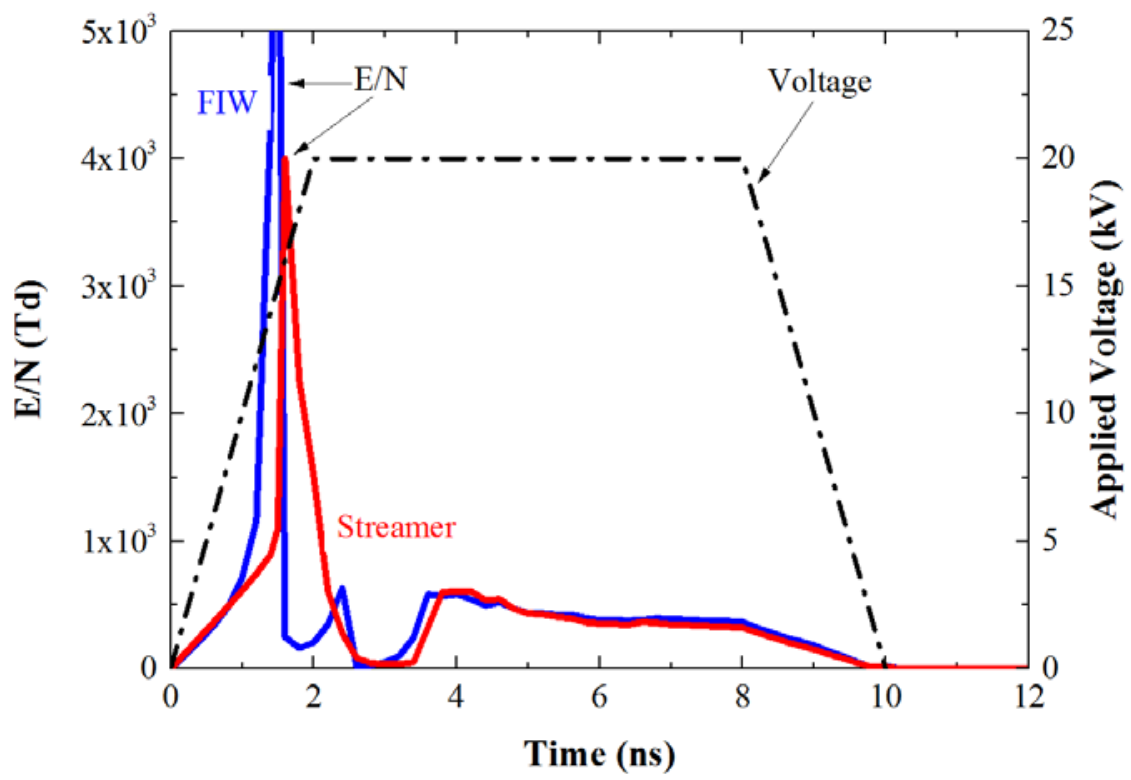

Figure 8. $E / N$ at the middle of the capillary tube $(r=0 \mathrm{~cm}, \mathrm{z}=1.5 \mathrm{~cm})$ for the two modes of discharge, FIW and streamer, in the nCD.

\subsubsection{Streamers in Tubes with Different Radii}

For a given stored energy in the pulsed power circuit, the radius of the discharge tube determines the deposited energy density in the nCD. However, given that streamers and ionization waves typically have radii that are functions of pressure, the radius of the discharge tube then may also determine the degree of physical confinement of the ionization wave. To investigate these scalings, simulations were conducted for $\mathrm{nCD}$ radii of $\mathrm{R}_{1}=0.75 \mathrm{~mm}$ (the experimental value), $\mathrm{R}_{2}=1.5 \mathrm{~mm}$ and $\mathrm{R}_{3}=3.75 \mathrm{~mm}$. In increasing the diameter of the discharge tube, the radii of the $\mathrm{LV}$ and HV electrodes were also increased in the same proportion. The LV electrode was grounded through a $100 \Omega$ resistor to enable the streamer mode. All other parameters remained the same. The spatial and axial evolution of electric field and electron density for different radii are in Figures 9 and 10 . 


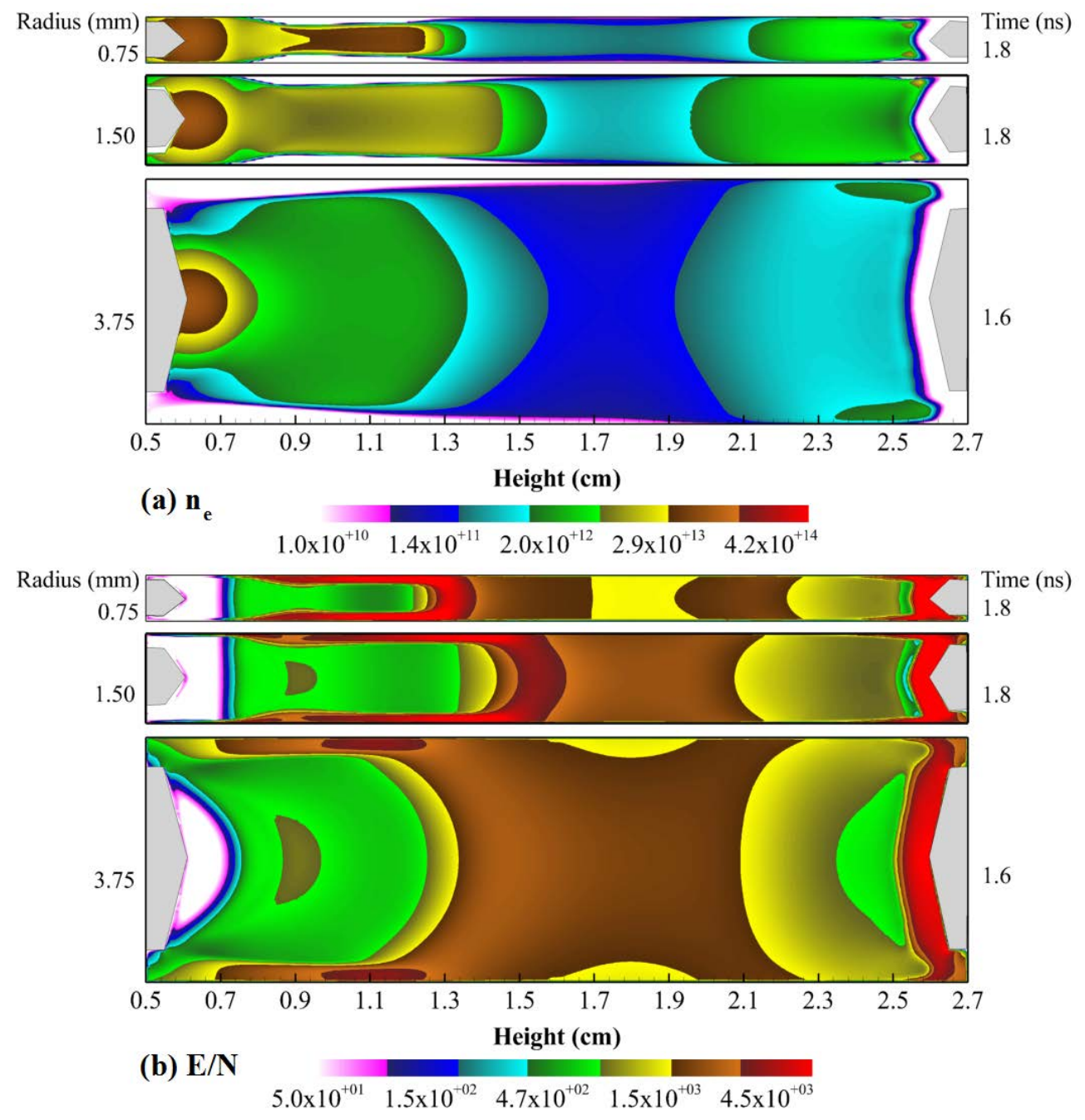

Figure 9. Plasma properties while varying the discharge tube radii $\left(\mathrm{R}_{1}=0.75 \mathrm{~mm}, \mathrm{R}_{2}=1.5 \mathrm{~mm}\right.$ and $\mathrm{R}_{3}=3.75$ mm. (a) electron density and (b) normalized electric field. (E/N)

For all radii, ionization waves start from both electrodes, propagate in opposite directions and then merge to form quasi-neutral plasma regions, consistent with the streamer mode. The initial intensity of the ionization waves decreases with increasing radius in part because the electric field enhancement at the tip of the electrodes decreases with increasing radii. The radius of the ionization head, $R_{s}$, increases with increasing discharge tube radii. $R_{s}$ is close to the radius of the narrow tube and so the streamer is confined by the discharge tube. This confinement leads to larger losses and so a larger $E / N$ is needed to sustain the ionization wave, while also producing larger energy deposition. With a larger discharge tube radius, confinement and losses decrease, charge separation in the head occurs over a larger region, leading to lower space charge density, lower $E / N$ and lower ionization rate. The larger losses for the smaller tube reduce the streamer propagation velocity. The increase in propagation speed, $\mathrm{v}_{s}$, with increasing radius is consistent with the scaling proposed in Ref [46],

$$
v_{S}=\frac{R_{S} v_{i}}{\ln \left(n_{e} / n_{0}\right)}
$$

where $v_{i}$ is the ionization frequency, $n_{e}$ is the electron density in the streamer and $n_{0}$ is the electron density at a distance $R_{S}$ from the ball-like surface of the streamer. The expression for streamer 
velocity, Eq. (15) is obtained from the ionization frequency, or the time of ionization growth $v_{\mathrm{i}}^{-1}$ [46], [47] assuming that the electron density stops increasing when the inverse time of the ionization growth is equal to the Maxwell relaxation time (the time for the EED to become a Maxwellian). In this work, the streamer is confined for the smaller tubes, and so $R_{S}$ is controlled by the tube radius. The ionization frequency $v_{i}$ is a function of $E / N$. The electric field at $1.8 \mathrm{~ns}$ for $\mathrm{R}_{1}=0.75 \mathrm{~mm}$ $(4500 \mathrm{Td})$ is about 1.5 times that of the larger radii $\mathrm{R}_{2}=1.5 \mathrm{~mm}$. The value of $\ln \left(n_{e} / n_{0}\right)$ decreases with the increase of tube radius (although $n_{e} / n_{0}$ are of the same magnitude). The combination of these factors produces a 1.1 to 1.3 times increase in streamer propagation velocity when doubling the tube radius.
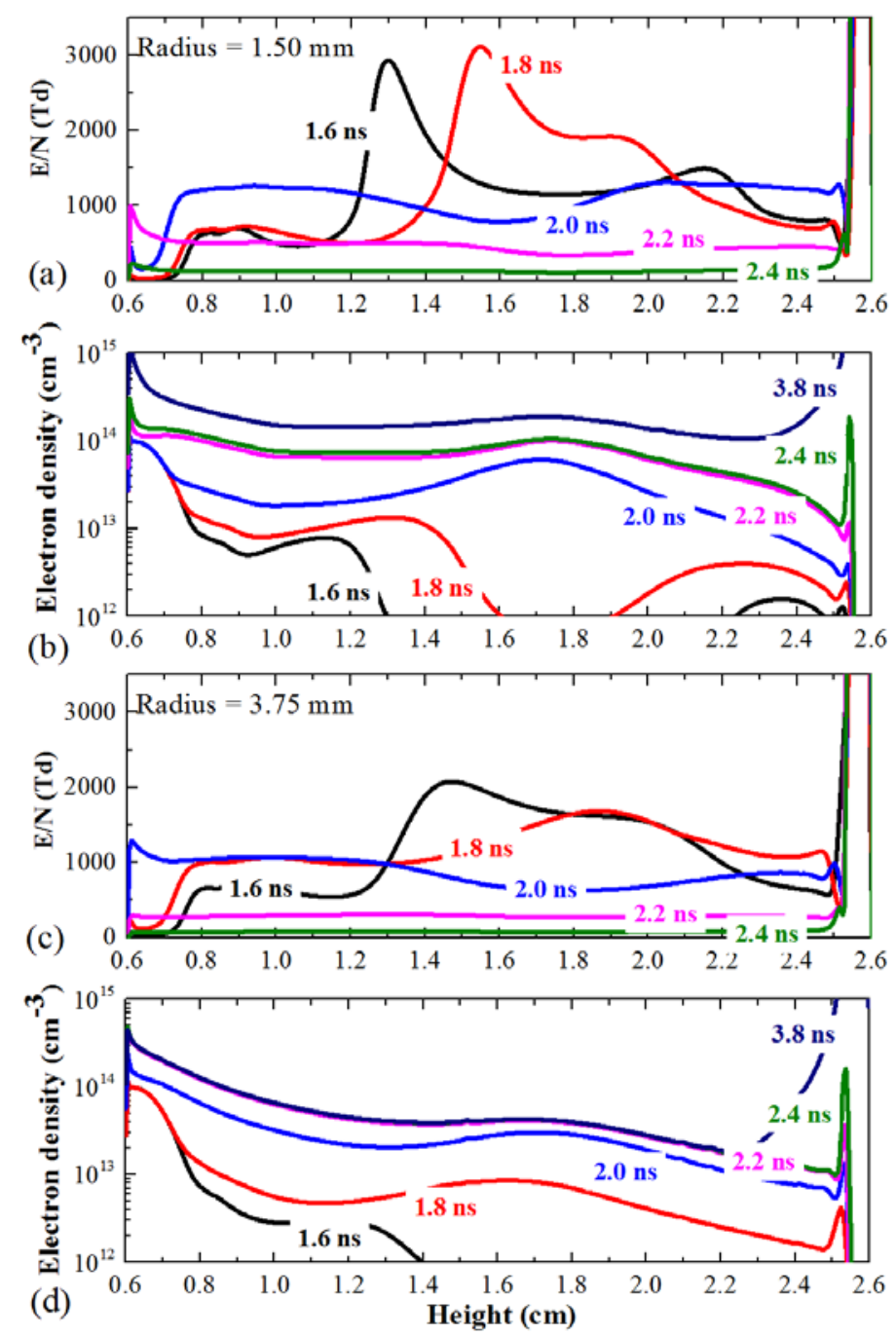

Figure 10. Axial distribution of electron density and electric field in tubes with radius of $R_{2}=1.5 \mathrm{~mm}$ and $\mathrm{R}_{3}=3.75 \mathrm{~mm}$. a) $E / N$ and b) electron density for $\mathrm{R}_{2}$; and c) $E / N$ and d) electron density for $\mathrm{R}_{3}$ ),

The radial distributions of electron density and ionization rates are functions of the discharge tube radius, as shown in Fig. 11. With increasing radius of the tube, the electron density in the plasma channel after transit of the ionization front decreases. For $\mathrm{R}_{1}=0.75 \mathrm{~mm}$, the electron density is maximum near the axis, $6 \times 10^{13} \mathrm{~cm}^{-3}$ with a relatively high $E / N$ in the head, $4500 \mathrm{Td}$. When the tube radius is doubled, $\mathrm{R}_{2}=1.5 \mathrm{~mm}$, the electron density decreases to $2 \times 10^{13} \mathrm{~cm}^{-3}$, and is more concentrated near the tube wall with lower $E / N$ in the ionization head, 2800Td. In the largest tube, 
$\mathrm{R}_{3}=3.75 \mathrm{~mm}$, a bulk plasma is formed, $2.5 \times 10^{12} \mathrm{~cm}^{-3}$, with little enhancement of electron density near the wall. The $E / N$ in the head is the weakest among all the radii, $2070 \mathrm{Td}$. For these conditions, the streamer is confined by the smallest tube, necessitating a larger $E / N$ to sustain the plasma and producing largest specific energy density. For the intermediate diameter, confinement is less while electric enhancement in crossing from the plasma to the quartz tube induces a surface ionization wave (SIW) [48] which enhances the electron density. The streamer radius is smaller than that of the largest tube, which then enables the streamer to propagate largely as a bulk ionization wave independent of the wall. Having said that, there is still a weak SIW that propagates in the large tube.
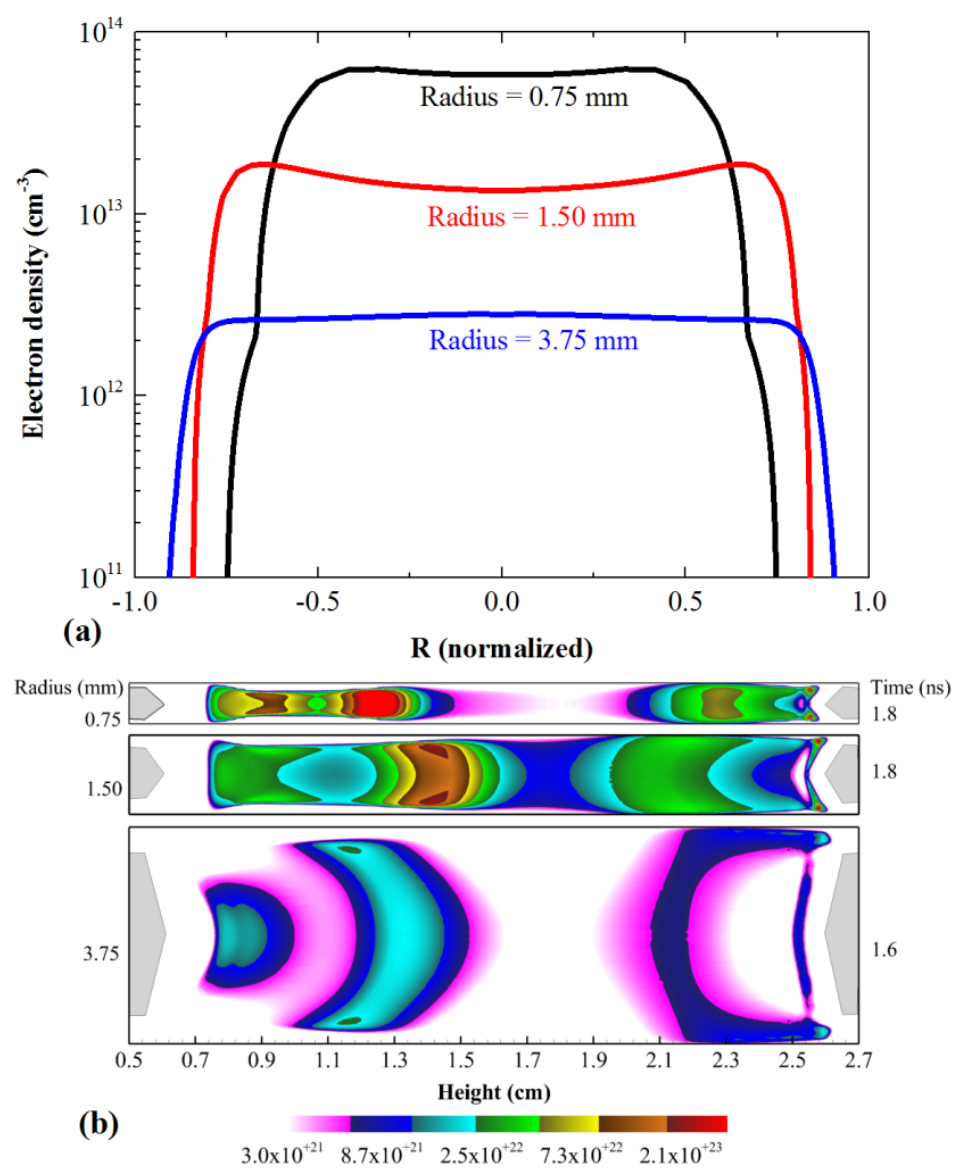

Figure 11. Plasma properties for different discharge tube radii. (a) Electron density as a function of radius at a height of $1.5 \mathrm{~cm}$. (b) Electron impact source in the ionization head. The data are shown at $1.8 \mathrm{~ns}$ for $\mathrm{R}_{1}$ and $\mathrm{R}_{2}$, and at 1.6 ns for $\mathrm{R}_{3}$

\subsection{Discharge and Early Afterglow: Energy Deposition and Species Evolution}

The influence of specific energy deposition in the plasma, $\omega_{\text {dep }}(\mathrm{eV} / \mathrm{molecule})$, on discharge properties was investigated by adjusting the resistance of the external circuit. For a given current, larger ballast resistors have a large voltage drop, leaving less voltage across the discharge tube and lower $\omega_{\text {dep. }}$. In this section, the dependence of electron density and density of $\mathrm{N}_{2}\left(\mathrm{C}^{3} \Pi_{\mathrm{u}}\right)$ ) will be discussed as a function of $\omega_{\text {dep. }}$.

\subsubsection{Electrical Characteristics and Specific Energy Deposition}

Once the FIW reaches the LV electrode, or the two streamers merge, the tube is filled with conductive plasma. Energy deposition dominantly occurs at this stage as the capacitance of the 
power supply delivers current through this conductive channel. With the simple circuit employed in the model, there is no inductance to slow the delivery of current and there is no drop in the power supply voltage due to depletion of its capacitance. The effects we discuss are then a result of voltage division between the plasma in the discharge tube and the ballast resistor. With a discharge tube radius of $\mathrm{R}_{1}=0.75$, to vary $\omega_{\text {dep }}$ the ballast resistor was varied from $20 \Omega$ to $12,700 \Omega$, which produced a range of $\omega_{\text {dep }}$ of $5 \mathrm{eV} /$ molecule $(20 \Omega)$ to $0.004 \mathrm{eV} /$ molecule $(12,700 \Omega)$, and a range of $E / N$ after breakdown of $600 \mathrm{Td}$ to $50 \mathrm{Td}$.
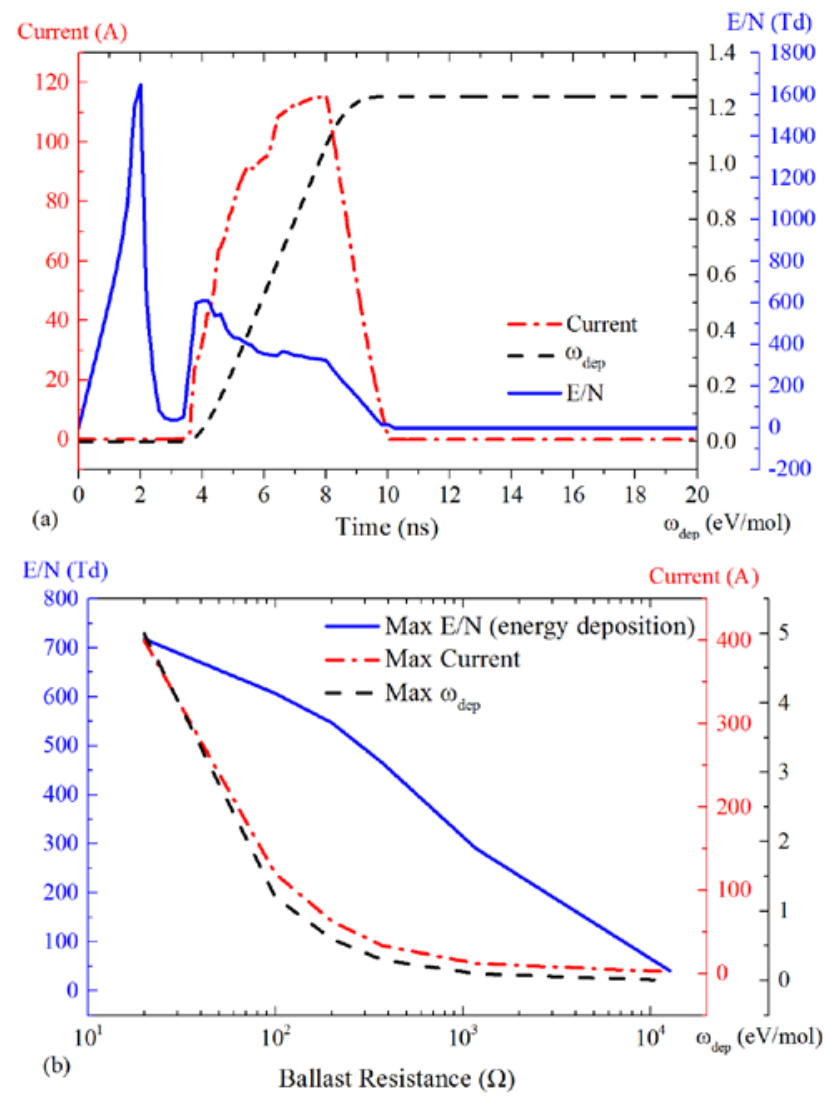

Figure 12. Calculated plasma properties of discharges in a short capillary tube with varying resistance in the external circuit. The tube radius is $R_{1}=0.75 \mathrm{~mm}$. (a) $\mathrm{E} / \mathrm{N}, \omega_{\text {dep }}$ and $\mathrm{E} / \mathrm{N}$ as a function of time for a $100 \Omega$ ballast resistor. (b) Maximum values of $\mathrm{E} / \mathrm{N}$, current and $\omega_{\text {dep }}$ as a function of ballast resistance.

The computed $E / N$, current and energy deposition are shown in Fig. 12(a) for a $100 \Omega$ ballast resistor. The maximum $E / N$ during the energy deposition stage after breakdown reaches $600 \mathrm{Td}$ while the current reaches $120 \mathrm{~A}$. The total deposited energy increases to $1.2 \mathrm{eV} / \mathrm{mol}$ before the current is terminated due at the end of the voltage pulse. Adjusting the ballast resistance leads to scaling of the properties in the energy deposition stage, as shown in Fig. 12(b). The maximum E/N during energy deposition falls below the nominal self-sustaining value ( 120 Td) when the ballast resistance is higher than $5.5 \mathrm{k} \Omega$. The change in energy deposition is closely related to the change in maximum current.

\subsubsection{Long-lived Plasma and Quenching of Excited Species}

An increase in specific energy deposition leads to an increase in the roles of collisions of electrons with short-lived electronically excited species, collisions of electronically excited species with neutrals, 
and collisions between electronically excited species. Not taking into account these processes can lead to significant errors in the analysis of experimental data, for example in deriving densities of species from actinometry and LIF/TALIF, as well as in numerical modeling. To demonstrate the importance of these processes at high $\omega_{\text {dep }}$, we use normalized values of the electron density and the density of $\mathrm{N}_{2}\left(\mathrm{C}^{3} \Pi_{\mathrm{u}}\right)$. (Normalized values are densities divided by their maximum value.) Both global modeling and experiments have shown that when operating at high $\omega_{\text {dep }}$ the decay in electron density in the early afterglow is slower than would be predicted solely by electron loss by attachment and recombination. These results also show that the density of $\mathrm{N}_{2}\left(\mathrm{C}^{3} \Pi_{\mathrm{u}}\right)$ decays faster than the rate given by its natural lifetime and quenching by the ground states of molecular nitrogen and oxygen.
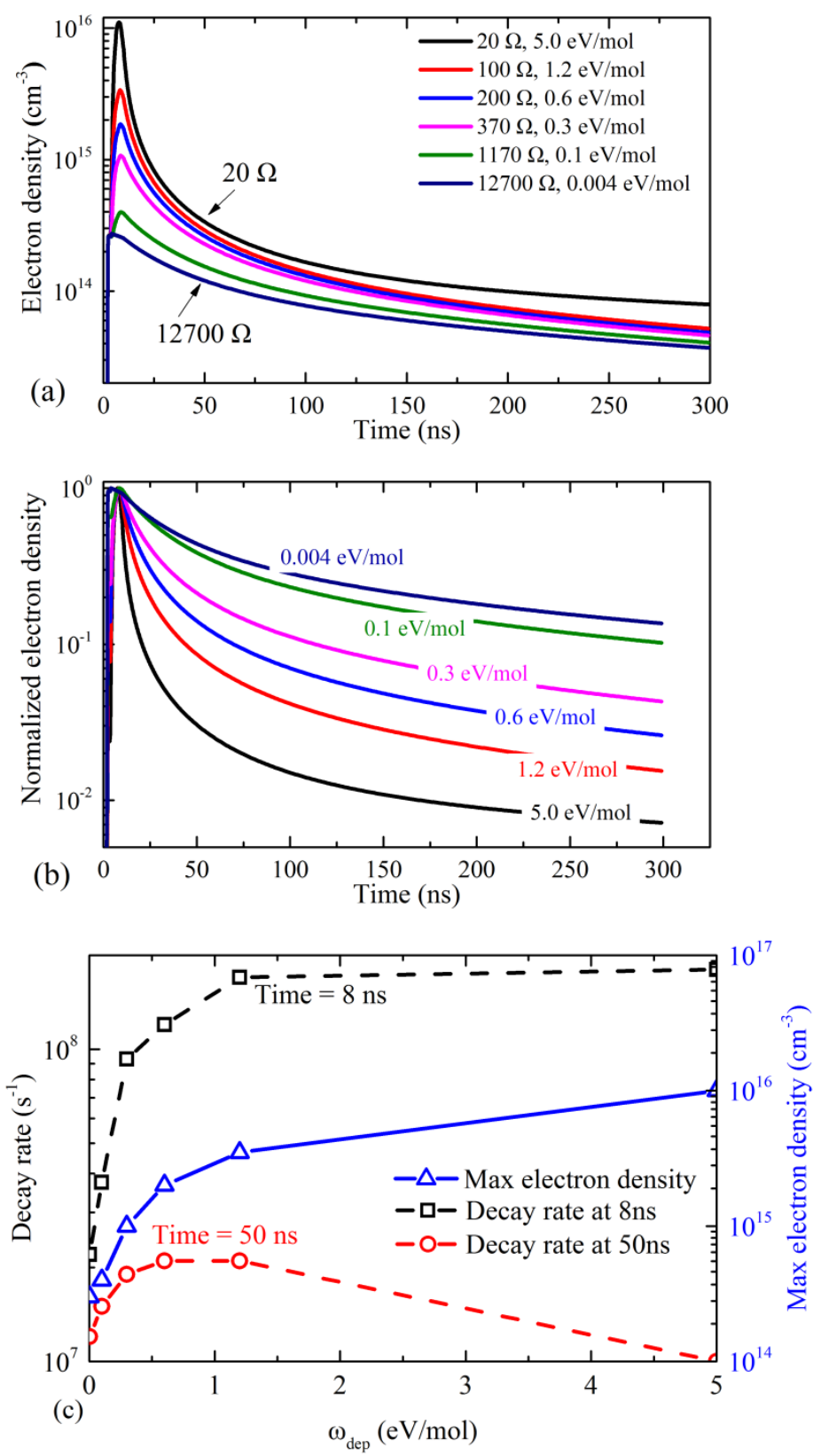

Figure 14. The time dependence of (a) absolute electron density and (b) normalized electron density for different specific energy depositions; and (c) the maximum electron density (blue line) and decay rate of electrons at 8 ns (black line) and at 50 ns (red line). 
The absolute and normalized densities of electrons are shown in Figure 14(a) and 14(b) as a function of time for different values of $\omega_{d e p}$. For $\mathrm{t}<10 \mathrm{~ns}$, electrons are mainly produced by direct electron impact ionization of ground state species,

$$
\begin{aligned}
& \mathrm{e}+\mathrm{N}_{2} \rightarrow \mathrm{N}_{2}^{+}+\mathrm{e}+\mathrm{e}, \\
& \mathrm{e}+\mathrm{O}_{2} \rightarrow \mathrm{O}_{2}^{+}+\mathrm{e}+\mathrm{e}
\end{aligned}
$$

The electrons are dominantly consumed by three body attachment, dissociative attachment and recombination (mainly with $\mathrm{O}_{2}^{+}$)

$$
\begin{aligned}
& \mathrm{e}+\mathrm{e}+\mathrm{O}_{2} \rightarrow \mathrm{O}_{2}^{-}+\mathrm{e} \\
& \mathrm{e}+\mathrm{O}_{2} \rightarrow \mathrm{O}+\mathrm{O}^{-} \\
& \mathrm{e}+\mathrm{O}_{2}^{+} \rightarrow \mathrm{O}+\mathrm{O}
\end{aligned}
$$

After the discharge pulse, the electron density decreases. The initial rate of decrease during the first 50 ns for $\omega_{\text {dep }}>0.1 \mathrm{eV} / \mathrm{mol}$ increases with $\omega_{\text {dep }}$, as shown in Figure 14(c). This fast initial decay is mainly due to the high electron and ion densities produced by the high $E / N$ during the discharge. With the rates of dissociative recombination (the dominant electron loss process) scaling with the square of the plasma density, higher rates of decay are expected at higher energy deposition. For example, dissociative recombination has a rate coefficient of approximately $10^{-7} \mathrm{~cm}^{3} \mathrm{~s}^{-1}$. With an initial electron and ion density of $10^{16} \mathrm{~cm}^{-3}$, the initial rate of decay is $10^{9} / \mathrm{s}$. With an initial plasma density of $10^{14} \mathrm{~cm}^{-3}$, the decay rate is $10^{7} / \mathrm{s}$. At the same time the densities of $\mathrm{N}_{2}\left(\mathrm{~A}^{3} \Sigma_{\mathrm{u}}^{+}\right)$and $\mathrm{N}_{2}\left(\mathrm{a}^{1} \Sigma_{\mathrm{u}}^{-}\right)$increase with increasing $\omega_{\text {dep }}$, producing ionization during the early afterglow by the associative ionization reactions in Eq. 14. For associative ionization rates coefficients of $10^{-11} \mathrm{~cm}^{3} \mathrm{~s}^{-1}$ and $\mathrm{N}_{2}\left(\mathrm{~A}^{3} \Sigma_{\mathrm{u}}^{+}\right)$and $\mathrm{N}_{2}\left(\mathrm{a}^{\prime 1} \Sigma_{\mathrm{u}}^{-}\right)$ densities of $10^{15} \mathrm{~cm}^{-3}$ for $\omega_{\text {dep }}=1.2 \mathrm{eV} / \mathrm{mol}$, rates of associative ionization are $10^{19} \mathrm{~cm}^{-3} \mathrm{~s}^{-1}$. These rates are smaller than the rates of dissociative recombination and attachment but do serve to slow down the decay of electron density. At $50 \mathrm{~ns}$, the combination of dissociative recombination and associative ionization leads to a transition region between $0.6 \mathrm{eV} / \mathrm{mol}$ and $1.2 \mathrm{eV} / \mathrm{mol}$ where the decay rate reaches a maximum of $2 \times 10^{7} / \mathrm{s}$.

Starting from $\mathrm{t}=50 \mathrm{~ns}$, the density of electrons decays with a slower rate. With the electron temperature at this time $T_{e}<0.2 \mathrm{eV}$, dissociative recombination is largely responsible for the rate of decay. The rate of decay for cases with higher $\omega_{\text {dep }}$, which was high in the early afterglow, decreases. In this time, associative ionization (see equations (14)) becomes an important electron source.

The mechanisms for production and loss of $\mathrm{N}_{2}\left(\mathrm{C}^{3} \Pi_{\mathrm{u}}\right)$ have already been discussed in prior studies, including the role of quenching by electrons in pure nitrogen [25]. The role of the quenching of $\mathrm{N}_{2}\left(\mathrm{C}^{3} \Pi_{\mathrm{u}}\right)$ by electrons was found to be important in interpreting the results of O-atom actinometry [34] for $\omega_{\text {dep }} \approx 1.0 \mathrm{eV} / \mathrm{mol}$.

The densities of $\mathrm{N}_{2}\left(\mathrm{C}^{3} \Pi_{\mathrm{u}}\right)$, absolute and normalized, are shown in Figure 15 as a function of time for different $\omega_{d e p}$. At low $\omega_{d e p}$, the decay is dominated by the quenching with oxygen molecules and radiative decay. For example, at $\omega_{d e p}=0.004 \mathrm{eV} / \mathrm{mol}$, the decay rate is $6.5 \times 10^{7} / \mathrm{s}$ with $\mathrm{N}_{2}\left(\mathrm{C}^{3} \Pi_{\mathrm{u}}\right)$ being $2 \times 10^{14} \mathrm{~cm}^{-3}$. The role of electron quenching can be seen at higher $\omega_{\text {dep }}$ (e.g. 5.0 $\mathrm{eV} / \mathrm{mol})$ despite the high $\mathrm{N}_{2}\left(\mathrm{C}^{3} \Pi_{\mathrm{u}}\right)$ densities that are also produced during the discharge, $5 \times 10^{16} \mathrm{~cm}^{-3}$. The higher density of $\mathrm{N}_{2}\left(\mathrm{C}^{3} \Pi_{\mathrm{u}}\right)$ decays faster in the afterglow, $4.6 \times 10^{8} / \mathrm{s}$, at high $\omega_{\text {dep }}$ due to the higher rate of electron collision quenching. Within 100 ns the density of $N_{2}\left(C^{3} \Pi_{u}\right)$ is lower than for discharges having smaller $\omega_{\text {dep }}$. 

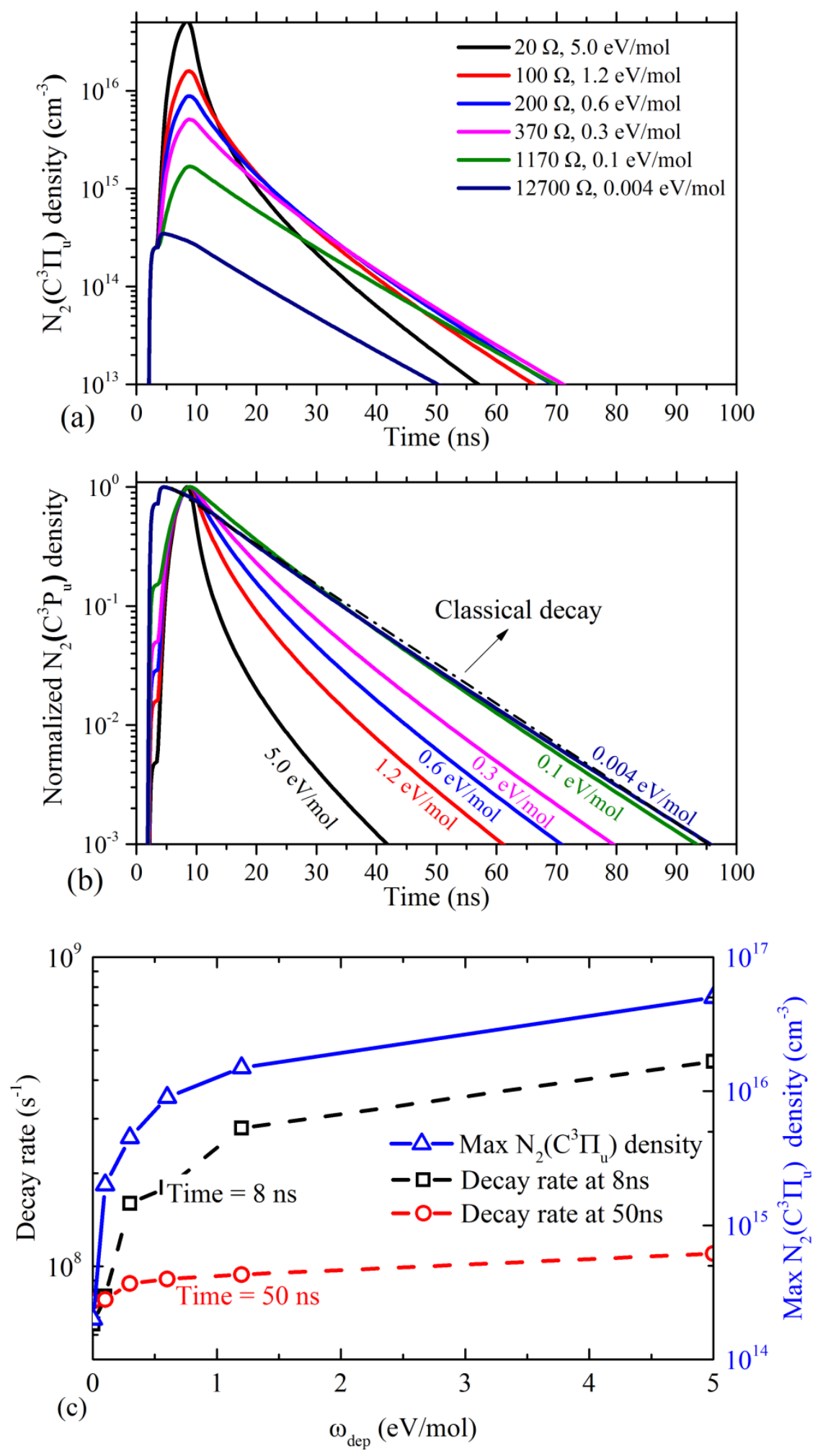

Figure 15. The temporal evolution of (a) absolute $\mathrm{N}_{2}\left(C^{3} \Pi_{\mathrm{u}}\right)$ density and (b) normalized $\mathrm{N}_{2}\left(\mathrm{C}^{3} \Pi_{\mathrm{u}}\right)$ density during the discharge and afterglow for different specific energy deposition; and (c) the maximum density of $\mathrm{N}_{2}\left(\mathrm{C}^{3} \Pi_{\mathrm{u}}\right)$ at $8 \mathrm{~ns}$ (blue line) and its decay rate at $8 \mathrm{~ns}$ (black line) and $50 \mathrm{~ns}$ (red line).

The normalized $\mathrm{N}_{2}\left(\mathrm{C}^{3} \Pi_{\mathrm{u}}\right)$ densities for different $\omega_{\text {dep }}$ (Figure 15(b)) show the onset of electron 
collisions quenching. For low $\omega_{\text {dep }}$, the decay rate for $\mathrm{N}_{2}\left(\mathrm{C}^{3} \Pi_{\mathrm{u}}\right)$ in the afterglow is essentially that given radiative decay and quenching by neutral molecules. For energy deposition increasing from $\omega_{\text {dep }}=0.1 \mathrm{eV} / \mathrm{mol}$, the density of $\mathrm{N}_{2}\left(\mathrm{C}^{3} \Pi_{\mathrm{u}}\right)$ increases to $2 \times 10^{15} \mathrm{~cm}^{-3}$, and the electron density reaches $4 \times 10^{14} \mathrm{~cm}^{-3}$. This increase in electron density coincides with the decay rate of $\mathrm{N}_{2}\left(\mathrm{C}^{3} \Pi_{\mathrm{u}}\right)$ in the afterglow increasing above that given by radiative decay and quenching by neutral molecules. The rate of electron collision quenching (rate constant $10^{-7} \mathrm{~cm}^{3} \mathrm{~s}^{-1}$ ) is $8 \times 10^{22} \mathrm{~cm}^{-3} \mathrm{~s}^{-1}$ while quenching by oxygen (rate constant $1.3 \times 10^{-10} \mathrm{~cm}^{3} \mathrm{~s}^{-1}$ ) is approximately $2.8 \times 10^{22} \mathrm{~cm}^{-3} \mathrm{~s}^{-1}$. Further increase in the specific energy deposition leads to higher electron densities and higher $\mathrm{N}_{2}\left(\mathrm{C}^{3} \Pi_{\mathrm{u}}\right)$ decay rates.

The kinetics of electrons and $\mathrm{N}_{2}\left(\mathrm{C}^{3} \Pi_{\mathrm{u}}\right)$ in the afterglow of the $\mathrm{nCD}$ is a sensitive function of the specific energy deposition $\omega_{\text {dep }}$. For energy deposition of $\omega_{\text {dep }} \leq 0.1 \mathrm{eV} / \mathrm{mol}$, decay rates are well described by the natural lifetime and quenching by neutral molecules. With increasing $\omega_{d e p}$, the rates of decay of the densities of electrons and $\mathrm{N}_{2}\left(\mathrm{C}^{3} \Pi_{\mathrm{u}}\right)$ significantly increase.

\subsubsection{Temporal-Spatial Evolution of Species}

After the tube is filled with plasma, the loss mechanisms for electrons and $N_{2}\left(C^{3} \Pi_{u}\right)$ lead to the redistribution of species in the afterglow. This redistribution of species has been previously observed and analyzed with a 1D kinetics model [25], [49].
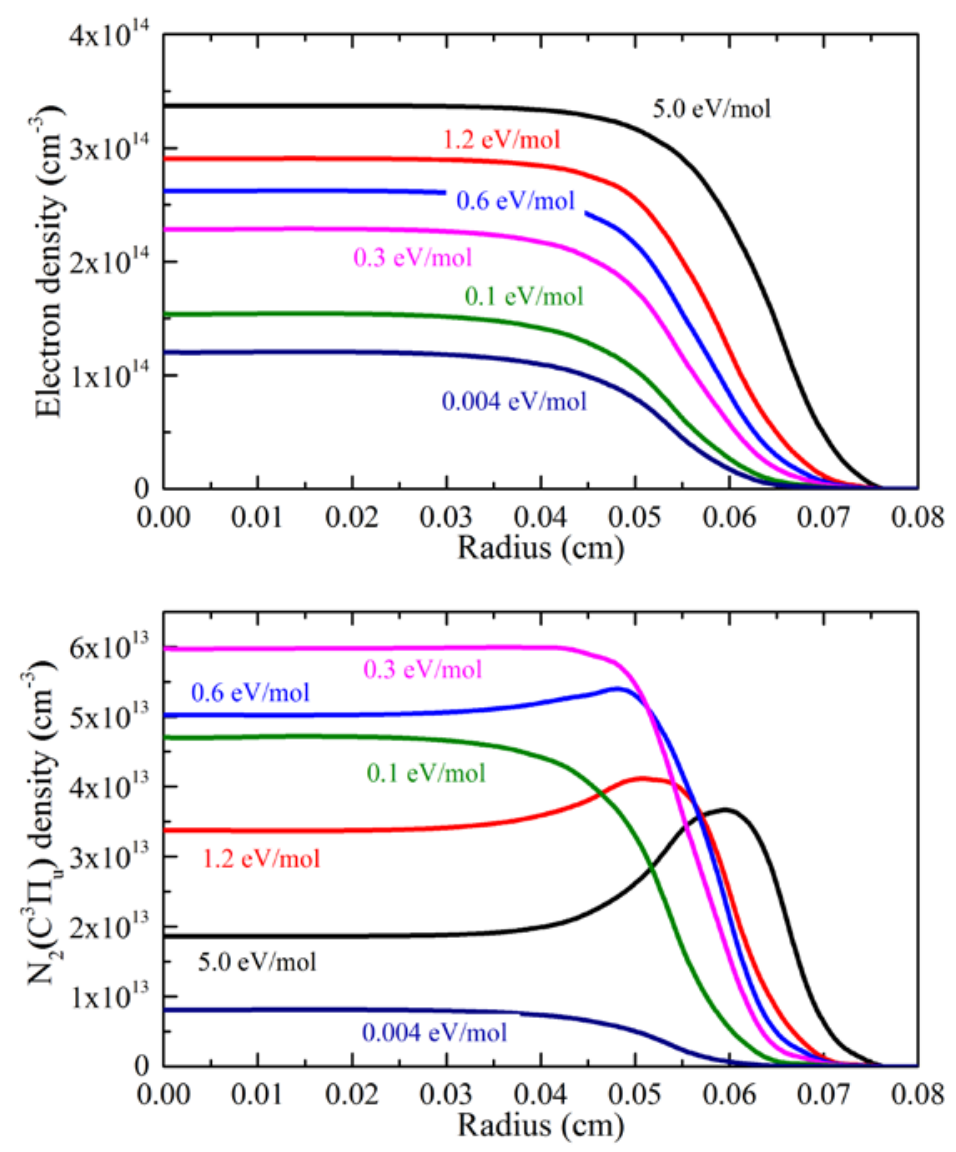

Figure 16. The radial distribution of (a) absolute electron density and (b) absolute $\mathrm{N}_{2}\left(\mathrm{C}^{3} \Pi_{\mathrm{u}}\right)$ density at $\mathrm{z}=1.5 \mathrm{~cm}$ and $\mathrm{t}=50 \mathrm{~ns}$.

The radial distributions of densities for different $\omega_{\text {dep }}$ in the afterglow at $t=50$ ns are shown in 
Figure 16(a) for electrons and in Figure 16(b) for $\mathrm{N}_{2}\left(\mathrm{C}^{3} \Pi_{\mathrm{u}}\right)$. The axial electron density scales with increasing $\omega_{\text {dep }}$, increasing from $1.5 \times 10^{14}$ at $\omega_{\text {dep }}=0.004 \mathrm{eV} / \mathrm{mol}$ to $3.5 \times 10^{14} \mathrm{~cm}^{-3}$ at $\omega_{\text {dep }}=5.0 \mathrm{eV} / \mathrm{mol}$. (The maximum densities during the discharge pulse span from $3 \times 10^{14} \mathrm{~cm}^{-3}$ to $1 \times 10^{16} \mathrm{~cm}^{-3}$.) The width of the high density column also increases, as discussed below.

The radial distributions of $\mathrm{N}_{2}\left(\mathrm{C}^{3} \Pi_{\mathrm{u}}\right)$ as a function of $\omega_{\text {dep }}$ at 50 ns are significantly different than those of electrons. For $\omega_{\text {dep }}$ up to $0.3 \mathrm{eV} / \mathrm{mol}$, the radial distribution of $\mathrm{N}_{2}\left(\mathrm{C}^{3} \Pi_{\mathrm{u}}\right)$ is similar to that of electrons, increasing with increasing $\omega_{\text {dep }}$, and having an on axis maximum. The density has a maximum of $6.0 \times 10^{13} \mathrm{~cm}^{-3}$ at $\omega_{d e p}=0.3 \mathrm{eV} / \mathrm{mol}$. With further increase in $\omega_{\text {dep }}$ there is an overall decrease in the density of $\mathrm{N}_{2}\left(\mathrm{C}^{3} \Pi_{\mathrm{u}}\right)$. The density decreases more rapidly on axis, resulting in a local maximum in density at increasingly larger radius. With an increase in the maximum electron density from $3 \times 10^{14} \mathrm{~cm}^{-3}$ to $1 \times 10^{16} \mathrm{~cm}^{-3}$ the rates of electron collision quenching of $\mathrm{N}_{2}\left(\mathrm{C}^{3} \Pi_{\mathrm{u}}\right)$ increase by a factor of 30. This increase in quenching is higher on axis where the electron density is maximum and lower at larger radius. These different rates of quenching produce a lower density of $\mathrm{N}_{2}\left(\mathrm{C}^{3} \Pi_{u}\right)$ in the center of the tube compared to the edge.

Normalized densities of electrons and $\mathrm{N}_{2}\left(\mathrm{C}^{3} \Pi_{\mathrm{u}}\right)$ as a function of radius at different times during the discharge and afterglow are shown in Figure 17 for $\omega_{\text {dep }}=0.004,0.5$ and $6 \mathrm{eV} / \mathrm{mol}$. The radial density profiles for electrons are nearly the same for all $\omega_{\text {dep }}$ at the end of the discharge at $10 \mathrm{~ns}$. In the afterglow, the overall radial distribution is the same with a plateau at small radius and a sharp drop near the edge. However, during the afterglow, the width of the plasma channel increases with increasing $\omega_{\text {dep }}$. The increasing width of the channel results from increasing rates of electron-ion recombination with increasing $\omega_{\text {dep }}$ which depresses the density at small radius where the absolute densities are initially largest. With increasing $\omega_{\text {dep }}$ there is an increase in the densities of $\mathrm{N}_{2}\left(\mathrm{~A}^{3} \Sigma_{\mathrm{u}}^{+}\right)$ and $\mathrm{N}_{2}\left(\mathrm{a}^{\prime 1} \Sigma_{\mathrm{u}}^{-}\right)$, which then augment densities at larger radius by associative ionization reactions.

At low $\omega_{\text {dep }}$ the radial distributions of $\mathrm{N}_{2}\left(\mathrm{C}^{3} \Pi_{\mathrm{u}}\right)$ mirror those of the electrons. Under these conditions, the radial optical emission of $\mathrm{N}_{2}\left(\mathrm{C}^{3} \Pi_{\mathrm{u}}\right)$ is a good indication of the distribution of electrons. With an increase of $\omega_{\text {dep }}, \mathrm{N}_{2}\left(\mathrm{C}^{3} \Pi_{\mathrm{u}}\right)$ is strongly quenched by electrons at radii within the plateau where the electron density is high. With further increases of $\omega_{\text {dep }}$, the quenching is stronger on axis, leading to a hollow radial distribution of $\mathrm{N}_{2}\left(\mathrm{C}^{3} \Pi_{\mathrm{u}}\right)$. The radius at which the profile of $\mathrm{N}_{2}\left(\mathrm{C}^{3} \Pi_{\mathrm{u}}\right)$ is maximum during the afterglow also increases with $\omega_{\text {dep }}$. At this time, the electron temperature is not high enough to collisionally produce $\mathrm{N}_{2}\left(\mathrm{C}^{3} \Pi_{\mathrm{u}}\right)$, and the time is too short for diffusion to significantly change the radial profile. The apparent expansion of the $N_{2}\left(C^{3} \Pi_{u}\right)$ channel during the afterglow is a result of electron collision quenching and energy pooling reactions with $\mathrm{N}_{2}\left(\mathrm{~A}^{3} \Sigma_{\mathrm{u}}^{+}\right)$at smaller radii.

The 2D distributions of electrons and $\mathrm{N}_{2}\left(\mathrm{C}^{3} \Pi_{\mathrm{u}}\right)$ at $\mathrm{t}=100$ ns are shown in Figure 18 for $\omega_{\text {dep }}=$ 0.004, 0.6 and $5 \mathrm{eV} / \mathrm{mol}$. The following features of the nCD during the afterglow generally occur throughout the plasma column: (i) the electron density increases with increasing $\omega_{d e p}$, (ii) the $\mathrm{N}_{2}\left(\mathrm{C}^{3} \Pi_{\mathrm{u}}\right)$ density increases for $\omega_{\text {dep }} \leq 0.6 \mathrm{eV} / \mathrm{mol}$ and decreases at higher $\omega_{\text {dep }}$, (iii) the radii of the electron and $\mathrm{N}_{2}\left(\mathrm{C}^{3} \Pi_{\mathrm{u}}\right)$ channels grow with increasing $\omega_{\text {dep }}$, and (iv) the radial profiles of the electron densities remain the same, while the radial distributions of the $\mathrm{N}_{2}\left(\mathrm{C}^{3} \Pi_{\mathrm{u}}\right)$ density evolve to be hollow with a higher $\mathrm{N}_{2}\left(\mathrm{C}^{3} \Pi_{\mathrm{u}}\right)$ density located closer to the wall. 

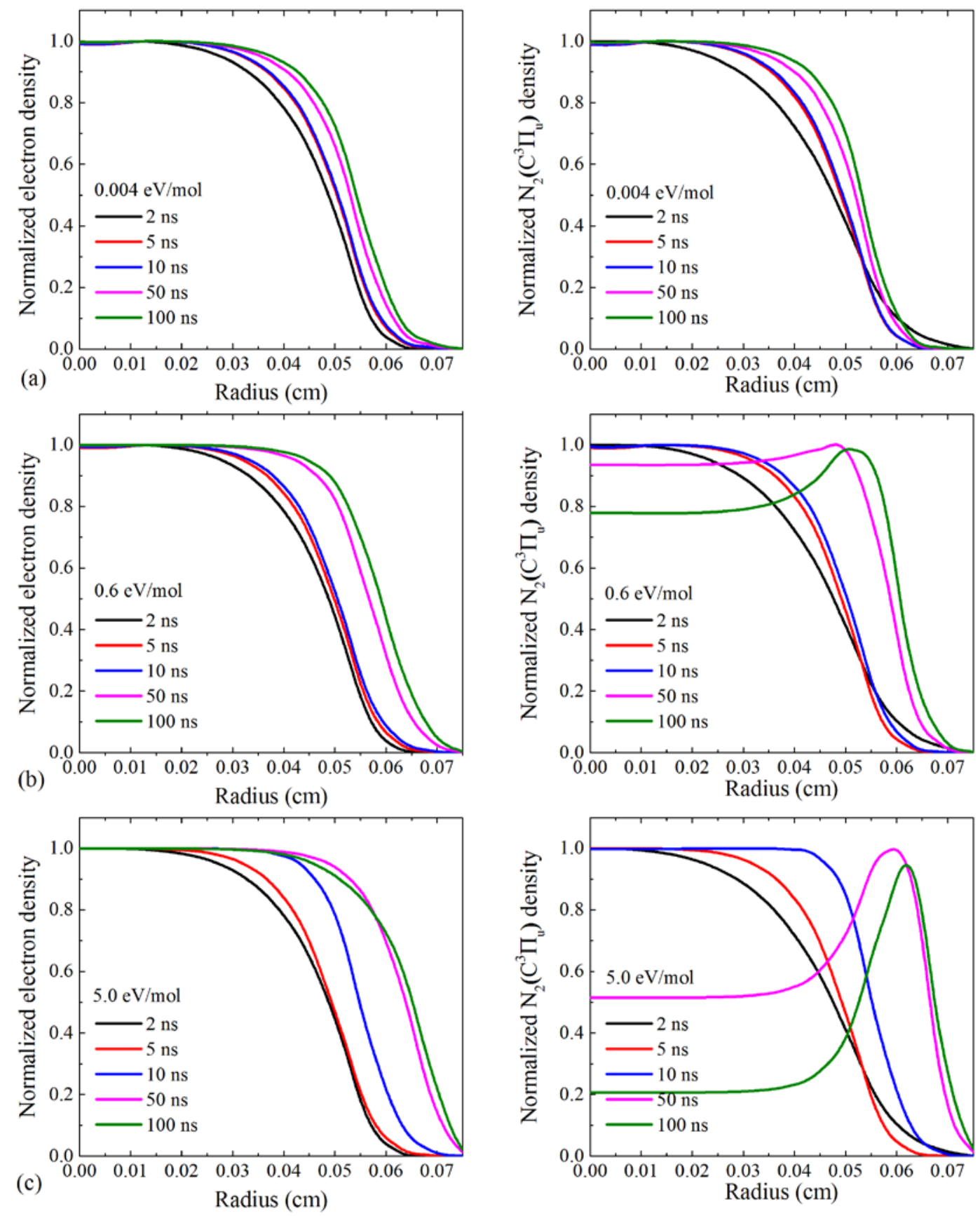

Figure 17. The normalized (left) electron density (left) and (right) $\mathrm{N}_{2}\left(\mathrm{C}^{3} \Pi_{\mathrm{u}}\right)$ density as a function of radius at different times at $\mathrm{z}=1.5 \mathrm{~cm}$ for specific energy depositions of (a) $0.004 \mathrm{eV} / \mathrm{mol}$, (b) $0.5 \mathrm{eV} / \mathrm{mol}$ and (c) $5.0 \mathrm{eV} / \mathrm{mol}$. 

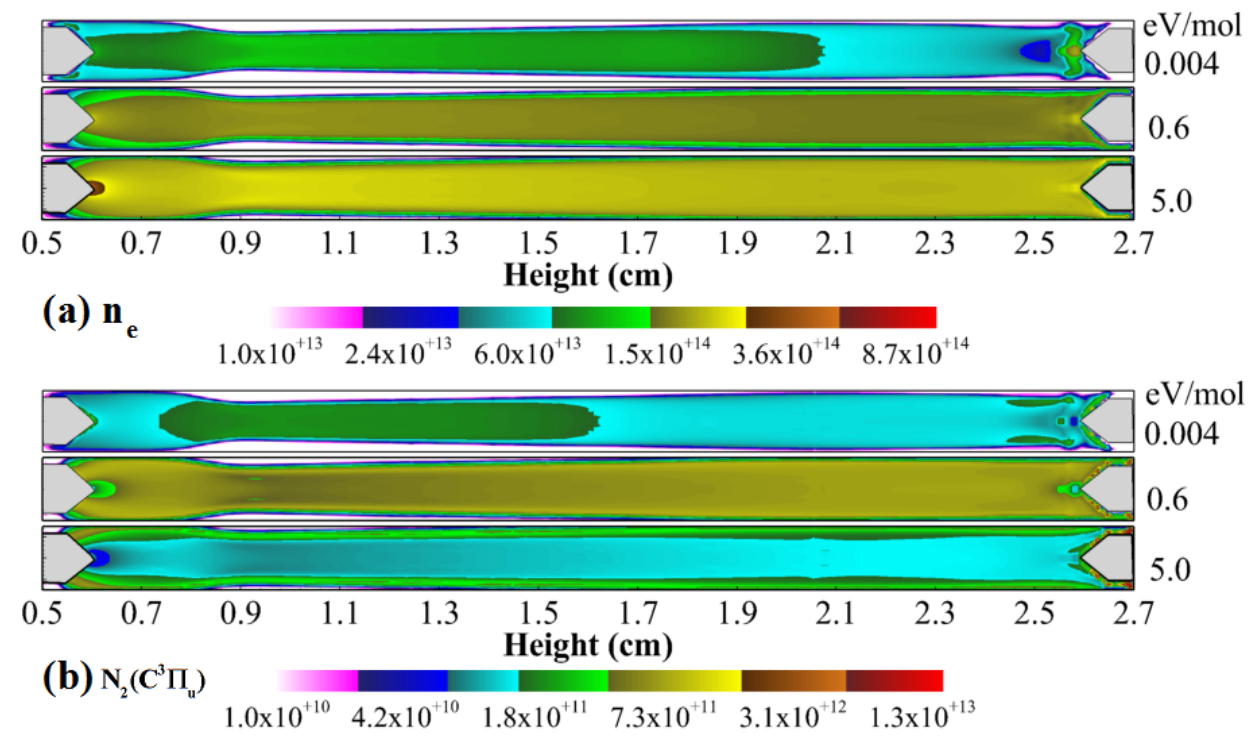

Figure 18. 2D distribution of (a) electrons and (b) $\mathrm{N}_{2}\left(C^{3} \Pi_{\mathrm{u}}\right)$ at $\mathrm{t}=100 \mathrm{~ns}$ for different specific energy depositions.

\section{Concluding Remarks}

The development of nanosecond capillary discharges in air at moderate pressure and different specific energy depositions was discussed using results from 2D numerical modeling. Propagation of ionization waves and filling of the discharge tube with plasma were investigated. Two modes of propagation were identified depending on whether the low voltage (LV) electrode was grounded through a resistor or was electrically floating. A fast ionization wave (FIW) mode occurred with a floating LV electrode and streamer mode occurred with a grounded electrode. In the streamer mode, two ionization waves starting at the high voltage (HV) and LV electrodes propagate towards each other and merge. In the FIW mode, a single ionization wave propagates from the HV electrode to the LV electrode with a higher $E / N$ in the head of the ionization wave and higher electron density in the channel. Despite these differences, a uniform distribution of electron density along the discharge tube is achieved in both propagation modes.

Radial distributions of the electron density are strong functions of the radius of the tube. At a tube radius of $\mathrm{R}_{3}=3.75 \mathrm{~mm}$, a bulk plasma was formed that was nearly uniformly distributed in the radial direction. At $\mathrm{R}_{2}=1.5 \mathrm{~mm}$, a higher electron density occurred near the tube wall, leaving a hollow region on axis. With further decrease of the radius to $\mathrm{R}_{1}=0.75 \mathrm{~mm}$, the peaks of electron density near the walls merged and a high electron density occurred on the axis of the capillary tube. The propagation velocity of the ionization wave increased with tube radius.

Based on the plasma distribution produced in the streamer mode, the kinetics in the early afterglow was modeled to investigate the behavior of selected species with respect to specific energy deposition, $\omega_{\text {dep }}$. The peak electron density during the voltage pulse increased with increasing $\omega_{\text {dep }}$. The decay rate of electron density increased with $\omega_{\text {dep }}$ immediately after the discharge due to this higher initial electron density that increased rates of recombination (both 2-body and-3 body). At $t \geq 50$ ns during the afterglow, the decay rate of electron density slows due to production of electrons by associative ionization. The importance of associative ionization in the afterglow increases with increasing $\omega_{\text {dep }}$. The radial profile of electrons stays nearly the same during the $300 \mathrm{~ns}$ of the discharge and early afterglow. 
The decay rate of $\mathrm{N}_{2}\left(\mathrm{C}^{3} \Pi_{\mathrm{u}}\right)$ during the afterglow increases with increasing $\omega_{\text {dep }}$. For $\omega_{\text {dep }}<$ $0.3 \mathrm{eV} / \mathrm{mol}$, the decay of $\mathrm{N}_{2}\left(\mathrm{C}^{3} \Pi_{\mathrm{u}}\right)$ during the afterglow is well described by its natural radiative lifetime and quenching by ground state molecules. The radial profile of $\mathrm{N}_{2}\left(\mathrm{C}^{3} \Pi_{\mathrm{u}}\right)$ closely resembles that for electrons. For $\omega_{\text {dep }} \geq 0.3 \mathrm{eV} / \mathrm{mol}$ quenching by electrons becomes important. This electron quenching, more rapid on axis where the electron density is higher, produces a hollow radial profile of $\mathrm{N}_{2}\left(\mathrm{C}^{3} \Pi_{\mathrm{u}}\right)$ with a minimum on axis and a maximum at larger radius. Due to this quenching with increasing $\omega_{\text {dep }}$, the density of $\mathrm{N}_{2}\left(\mathrm{C}^{3} \Pi_{\mathrm{u}}\right)$ during the afterglow increases with specific energy deposition if $\omega_{\text {dep }} \leq 0.3 \mathrm{eV} / \mathrm{mol}$ and decreases if $\omega_{\text {dep }} \geq 0.6 \mathrm{eV} / \mathrm{mol}$.

Taking into account excited state chemistry and electron quenching is critical in nanosecond discharges with high specific energy deposition. On the time scale of tens to hundreds of nanoseconds molecular electronic states with high threshold energies may have relatively high densities and dominate plasma chemistry. These interactions, and electron collision quenching, should be taken into account in optical diagnostics as additional losses (or sources).

\section{Acknowledgements}

The work was partially supported by French National Agency, ANR (ASPEN Project, ANR-16-CE30-0004-01), by LabEx Plas@Par, as part of the "Investments for the Future” French National Program ANR-11-IDEX-0004-02, by Linked International Laboratory LIA KaPPA (France-Russia) and by the National Natural Science Foundation of China (51907204). The participation of M. Kushner supported by the US National Science Foundation (PHY-1902878) and the U.S. Department of Energy, Office of Sciences Fusion Energy Sciences under Award Nos. DE-SC000319 and DE-SC0020232. The authors are thankful to Dr. Nikolay Popov for numerous discussions concerning kinetics of the discharge at high specific delivered energy. 


\section{References}

[1] A. Y. Starikovskii, A. A. Nikipelov, M. M. Nudnova, and D. V Roupassov, "SDBD plasma actuator with nanosecond pulse-periodic discharge,” Plasma Sources Sci. Technol., vol. 18, no. 3, p. 34015, 2009.

[2] K. Takashima, Z. Yin, and I. V. Adamovich, "Measurements and kinetic modeling of energy coupling in volume and surface nanosecond pulse discharges,” Plasma Sources Sci. Technol., vol. 22, no. 1, p. 15013, 2013.

[3] D. L. Rusterholtz, D. A. Lacoste, G. D. Stancu, D. Z. Pai, and C. O. Laux, "Ultrafast heating and oxygen dissociation in atmospheric pressure air by nanosecond repetitively pulsed discharges,” J. Phys. D. Appl. Phys., vol. 46, no. 46, p. 464010, 2013.

[4] E. I. Mintoussov, S. J. Pendleton, F. G. Gerbault, N. A. Popov, and S. M. Starikovskaia, "Fast gas heating in nitrogen-oxygen discharge plasma: II. Energy exchange in the afterglow of a volume nanosecond discharge at moderate pressures,” J. Phys. D. Appl. Phys., vol. 44, no. 28, p. 285202, 2011.

[5] Y. Zhu, Y. Wu, W. Cui, Y. Li, and M. Jia, "Numerical investigation of energy transfer for fast gas heating in an atmospheric nanosecond-pulsed DBD under different negative slopes," $J$. Phys. D. Appl. Phys., vol. 46, no. 49, p. 495205, 2013.

[6] N. Popov, "Fast gas heating initiated by pulsed nanosecond discharge in atmospheric pressure air,” 51 $1^{\text {st }}$ AIAA Aerospace Sciences Meeting, Grapevine, TX, Jan. 2013. Paper AIAA 2013-1052.

[7] D. Z. Pai, D. A. Lacoste, and C. O. Laux, "Nanosecond repetitively pulsed discharges in air at atmospheric pressure-the spark regime,” Plasma Sources Sci. Technol., vol. 19, no. 6, p. 65015, 2010.

[8] G. Fridman, G. Friedman, A. Gutsol, A. B. Shekhter, V. N. Vasilets, and A. Fridman, “Applied plasma medicine,” Plasma Process. Polym., vol. 5, no. 6, pp. 503-533, 2008.

[9] M. G. Kong et al., "Plasma medicine: An introductory review," New J. Phys., vol. 11, no. 11, p. 115012, 2009.

[10] D. Dobrynin, G. Fridman, G. Friedman, and A. Fridman, "Physical and biological mechanisms of direct plasma interaction with living tissue,” New J. Phys., vol. 11, no. 11, p. 115020, 2009.

[11] J. Heinlin et al., "Plasma medicine: possible applications in dermatology," JDDG J. der Dtsch. Dermatologischen Gesellschaft, vol. 8, no. 12, pp. 968-976, 2010.

[12] M. Laroussi, "From killing bacteria to destroying cancer cells: 20 years of plasma medicine," Plasma Process. Polym., vol. 11, no. 12, pp. 1138-1141, 2014.

[13] C. Douat, G. Bauville, M. Fleury, M. Laroussi, and V. Puech, "Dynamics of colliding microplasma jets,” Plasma Sources Sci. Technol., vol. 21, no. 3, 2012.

[14] K. Song, G. Li, and Y. Ma, "A review on the selective apoptotic effect of nonthermal atmospheric-pressure plasma on cancer cells,” Plasma Med., vol. 4, no. 1-4, 2014.

[15] D. B. Graves, "Mechanisms of Plasma Medicine: Coupling Plasma Physics, Biochemistry, and Biology,” IEEE Trans. Radiat. Plasma Med. Sci., vol. 1, no. 4, pp. 281-292, 2017.

[16] S. Starikovskaia, A. Y. Starikovskii, and D. Zatsepin, "Hydrogen oxidation in a stoichiometric hydrogen-air mixture in the fast ionization wave," Combustion Theory and Modelling, vol. 5, no. 1, pp. 97-129, 2001.

[17] S. Starikovskaia, K. Allegraud, O. Guaitella, I. Kosarev, E. Mintusov, S. J. Pendleton, N. Popov, P. Sagulenko, and A. Rousseau, "Surface discharges: possible applications for plas- 
ma-assisted ignition and electric field measurements," $48^{\text {th }}$ AIAA Aerospace Sciences Meeting, Orlando, Florida, January 2010. Paper AIAA 2010-1587.

[18] A. Starikovskiy, N. Aleksandrov, and A. Rakitin, "Plasma-assisted ignition and deflagration-to-detonation transition,” Philos. Trans. R. Soc. A Math. Phys. Eng. Sci., vol. 370, no. 1960, pp. 740-773, 2012.

[19] A. Starikovskiy and N Aleksandrov, "Plasma-assisted ignition and combustion", Progress in Energy and Combustion Science, vol. 39, pp.61-110, 2013.

[20] Y. Ju, W. Sun, and Y. J. and W. Sun, "Plasma assisted combustion: Dynamics and chemistry,” Prog. Energy Combust. Sci., vol. 48, no. 3, pp. 21-83, 2015.

[21] S. B. Leonov, I. V. Adamovich, and V. R. Soloviev, "Dynamics of near-surface electric discharges and mechanisms of their interaction with the airflow," Plasma Sources Sci. Technol., vol. 25, no. 6, p. 63001, 2016.

[22] K. Takashima et al., "Experimental and modeling analysis of fast ionization wave discharge propagation in a rectangular geometry,” Phys. Plasmas, vol. 18, no. 8, p. 83505, 2011.

[23] N. B. Anikin, S. M. Starikovskaia, and A. Y. Starikovskii, "Study of the oxidation of alkanes in their mixtures with oxygen and air under the action of a pulsed volume nanosecond discharge," Plasma Phys. Reports, vol. 30, no. 12, pp. 1028-1042, 2004.

[24] N. Lepikhin, A. Klochko, M. Edwards, N. Popov, and S. Starikovskaia, “Capillary nanosecond discharges as a tool for the measurement of quenching coefficients at high specific energy deposition,” Proc. 53rd AIAA Aerosp. Sci. Meet., no. January, pp. 5-9, 2015.

[25] N. D. Lepikhin, A. V. Klochko, N. A. Popov, and S. M. Starikovskaia, "Long-lived plasma and fast quenching of $\mathrm{N} 2(\mathrm{C} 3 \Pi \mathrm{u})$ by electrons in the afterglow of a nanosecond capillary discharge in nitrogen,” Plasma Sources Sci. Technol., vol. 25, no. 4, p. 45003, 2016.

[26] A. V Klochko et al., "Experimental and numerical study of fast gas heating and $\mathrm{O}$ atom production in a capillary nanosecond discharge,” 52nd Aerosp. Sci. Meet., no. January, pp. 1-17, 2014.

[27] T. L. Chng, A. Brisset, P. Jeanney, S. M. Starikovskaia, I. V. Adamovich, and P. Tardiveau, "Electric field evolution in a diffuse ionization wave nanosecond pulse discharge in atmospheric pressure air,” Plasma Sources Sci. Technol., vol. 28, no. 9, 2019.

[28] T. S. Bangdou Huang, Cheng Zhang, Igor Adamovich, Yuri Akishev, "Surface ionization wave propagation in the nanosecond pulsed surface dielectric barrier discharge: the influence of dielectric material and pulse repetition rate,” Plasma Sources Sci. Technol., vol. 10, pp. 2240822418, 2020.

[29] Z. Xiong, E. Robert, V. Sarron, J.-M. M. Pouvesle, and M. J. Kushner, “Dynamics of ionization wave splitting and merging of atmospheric-pressure plasmas in branched dielectric tubes and channels,” J. Phys. D. Appl. Phys., vol. 45, no. 27, p. 275201, 2012.

[30] J. Jansky, F. Tholin, Z. Bonaventura, and A. Bourdon, "Simulation of the discharge propagation in a capillary tube in air at atmospheric pressure," Journal of Physics D: Applied Physics, vol. 43, no. 39, p. 395201, 2010.

[31] A. V. Klochko, S. M. Starikovskaia, Z. Xiong, and M. J. Kushner, "Investigation of capillary nanosecond discharges in air at moderate pressure: comparison of experiments and \{2D \} numerical modelling,” J. Phys. D. Appl. Phys., vol. 47, no. 36, p. 365202, 2014.

[32] A. V. Klochko, J. Lemainque, J. P. Booth, and S. M. Starikovskaia, "TALIF measurements of oxygen atom density in the afterglow of a capillary,” Plasma Sources Sci. Technol., vol. 24, no. 


\section{2, p. 25010, 2015.}

[33] N. A. Popov, "Pulsed nanosecond discharge in air at high specific deposited energy: fast gas heating and active particle production,” Plasma Sources Sci. Technol., vol. 25, no. 4, p. 44003, 2016.

[34] Y. Zhu, N. D. Lepikhin, I. S. Orel, A. Salmon, A. V. Klochko, and S. M. Starikovskaia, “Optical actinometry of O-atoms in pulsed nanosecond capillary discharge: Peculiarities of kinetics at high specific deposited energy,” Plasma Sources Sci. Technol., vol. 27, no. 7, p. 75020, 2018.

[35] S. A. Norberg, E. Johnsen, and M. J. Kushner, "Formation of reactive oxygen and nitrogen species by repetitive negatively pulsed helium atmospheric pressure plasma jets propagating into humid air,” Plasma Sources Science and Technology, vol. 24, no. 3, p. 035026, 2015.

[36] M. Mitchner and C. H. Kruger, "Partially ionized gases,” (New York: Wiley, 1973), Ch. 12.

[37] J. Dutton, “A survey of electron swarm data,” J. Phys. Chem. Ref. Data, vol. 4, no. 3, pp. 577856, 1975.

[38] H. Kucukarpaci and J. Lucas, "Simulation of electron swarm parameters in carbon dioxide and nitrogen for high E/N,” Journal of Physics D: Applied Physics, vol. 12, no. 12, p. 2123, 1979.

[39] J. Fletcher and I. Reid, "The transport parameters of an electron swarm in nitrogen at elevated E/N,” Journal of Physics D: Applied Physics, vol. 13, no. 12, p. 2275, 1980.

[40] C. R. Rao and G. G. Raju, "Growth of ionization currents in dry air at high values of $E / N$," Journal of Physics D: Applied Physics, vol. 4, no. 4, p. 494, 1971.

[41] J. Moruzzi and D. Price, "Ionization, attachment and detachment in air and air-CO2 mixtures," Journal of Physics D: Applied Physics, vol. 7, no. 10, p. 1434, 1974.

[42] A. V Phelps and L. C. Pitchford, “Anisotropic scattering of electrons by $\left\{\$ \backslash r m\left\{N \_2\right\} \$\right\}$ and its effect on electron transport,” Phys. Rev. A, vol. 31, no. 5, p. 2932, 1985.

[43] J. Bacri and A. Medani, "Electron diatomic molecule weighted total cross section calculation: Iii. main inelastic processes for $\mathrm{N}_{2}$ and $\mathrm{N}^{+}$," Physica B, vol. 112, no. 1, pp. 101-118, 1982.

[44] J. W. Gallagher, C. E. Brion, J. A. R. Samson, P. W. Langhoff, and J. A. R. S. and P. W. L. J W |Gallagher, C E Brion, "Absolute Cross Sections for Molecular Photoabsorption, Partial Photoionization and Ionic Photofragmentation Processes," J. Phys. Chem. Ref. data, vol. 17, no. 1, pp. 9-153, 1987.

[45] S. Pancheshnyi, M. Nudnova, and A. Starikovskii, "Development of a cathode-directed streamer discharge in air at different pressures: experiment and comparison with direct numerical simulation,” Phys. Rev. E, vol. 71, no. 1, p. 16407, 2005.

[46] A. Kulikovsky, "Positive streamer between parallel plate electrodes in atmospheric pressure air,” Journal of physics D: Applied physics, vol. 30, no. 3, p. 441, 1997.

[47] M. Dyakonov and V. Kachorovskii, "Theory of streamer discharge in semiconductors," Sov. Phys. JETP, vol. 67, no. 5, pp. 1049-1054, 1988.

[48] V. Pedro, F. Pechereau, and A. Bourdon, "Numerical study on the time evolutions of the electric field in helium plasma jets with positive and negative polarities,” Plasma Sources Sci. Technol., vol. 27, no. 2, p. 025007, 2018.

[49] N. D. Lepikhin, N. A. Popov, and S. M. Starikovskaia, "Fast gas heating and radial distribution of active species in nanosecond capillary discharge in pure nitrogen and N2:O2mixtures," Plasma Sources Sci. Technol., vol. 27, no. 5, 2018. 